\title{
A Domain Decomposition Approach for Coupled Modelling of Nonlinear Soil-Structure Interaction
}

\author{
H. Zolghadr Jahromi, B.A. Izzuddin, L. Zdravkovic
}

Department of Civil and Environmental Engineering, Imperial College London London SW7 2AZ, United Kingdom

\begin{abstract}
This paper proposes a new approach for modelling coupled nonlinear soil-structure interaction problems by domain decomposition. It is assumed that the soil-structure coupled system is physically partitioned into independently modelled soil and structure sub-domains. A coupling procedure based on the sequential iterative Dirichlet-Neumann coupling algorithm is presented, which utilizes the condensed tangent stiffness matrices at the soil-structure interface to ensure and accelerate convergence to compatibility in successive update of the boundary conditions. As a special case of the proposed approach, the condensed tangent stiffness matrix is approximated via reduced order models of the partitioned sub-domains throughout the coupling iterations. A simulation environment has been developed, utilizing discipline-oriented solvers for nonlinear structural and geotechnical analysis, and is discussed. This tool is used to demonstrate the applicability of the presented coupling algorithm in modelling nonlinear soilstructure interaction problems, highlighting its relative merits compared to interface relaxation algorithms. Furthermore, the developed tool is employed for a case study involving nonlinear soil-structure interaction analysis between a plane frame and soil subjected to ground excavation, where the computational and modelling benefits of the proposed approach and the developed tool are clearly established.
\end{abstract}

Keywords: nonlinear soil-structure interaction, domain decomposition, iterative coupling, interface relaxation, reduced order model, condensed tangent stiffness matrix. 


\section{Introduction}

Modelling of soil-structure interaction involves significant interplay between the soil and the structure, which demands an integrated interdisciplinary computational model combining the features of both structural and geotechnical modelling. In principle, there are two possible procedures for treating soil-structure interaction problems, namely: direct (monolithic / simultaneous) and domain decomposition (partitioning) techniques $[1,2]$. In the monolithic treatment of soil-structure interaction problems, the solution of the complete system of equations of the coupled problem is attenuated in one analysis scheme. Alternatively, in the partitioned treatment, the partitioned sub-domains, namely soil and structure, are computationally treated as isolated entities, and the response of the coupled system is calculated using already developed soil and structural solvers. Although coupled modelling of soil-structure interaction problems may be achieved using a monolithic treatment, a partitioned treatment with different partitioned subdomains modelled as separate computational entities, amongst which interaction effects are exchanged, can offer major benefits in the context of nonlinear soil-structure interaction. Such benefits include i) allowing field-specific discretisation and solution procedures that have proven performance for each partitioned sub-domain, ii) facilitating the reuse of existing nonlinear analysis solvers with all the resource savings that this brings, and iii) enabling parallel computations through problem partitioning [35]. In this respect, this work is primarily motivated by the lack of sophisticated monolithic tools for modelling nonlinear soil-structure interaction problems. Recognising the existence of advanced tools for nonlinear analysis of structure and soil, in isolation, the partitioned approach is adopted as a framework for coupling fieldspecific tools with minimal intrusion. Accordingly, in adopting the partitioned treatment, the focus is on providing an advanced capability for modelling nonlinear soilstructure interaction with existing field-specific codes rather than on achieving superior computational performance in comparison with the monolithic treatment.

Partitioned analysis is mainly carried out by using staggered [6-8] or iterative substructuring [9] methods. In the staggered approach, which is applicable to transient dynamic analysis only, the governing equations of the partitioned sub-domains are solved independently at each time step using predicted boundary conditions at the interface (either force or displacement), obtained from previous time step(s) by a 
predictor. Example applications of the staggered approach to soil-structure interaction analysis are those of Rizos and Wang [10] and O'Brien and Rizos [11]. The authors developed a partitioned method for soil-structure interaction analysis through a staggered time-marching scheme, where a standard Finite Element Method (FEM) model, representing the structure domain, was coupled to a Boundary Element Method (BEM) model representing the soil domain as an elastic half-space.

However, the staggered approach should be used with great care, since its stability is often conditional, and the accuracy can depend on the choice of unrealistically small time steps; moreover, it can only be applied to dynamic problems.

On the other hand, iterative coupling algorithms are stable and accurate for a wider range of time-step size and applicable to both static and dynamic problems, which is mainly achieved by introducing corrective iterations in the staggered approach, hence the name iterative methods. Frequently, a relaxation of the iteratively updated boundary conditions is augmented to the iterative coupling algorithms in order to improve the convergence characteristics, hence the term interface relaxation [12-14]. Example applications of the relaxation scheme to soil-structure interaction analysis can be found in the work by Hagen and Estorff [15], concentrating on transient dynamic investigation of 3D dam-reservoir-soil using iterative coupling procedure, and in a more recent application by Francois et al. [16], in which the interface relaxation iterative coupling technique is applied to calculate the response of a structure due to traffic induced vibrations. Despite the significant potential benefits of interface relaxation iterative coupling algorithms (such as their simplicity of implementation and undemanding update of boundary conditions in corrective iterations), there are several issues regarding the applicability of such techniques, namely: i) determination of the range of suitable relaxation parameters for the specific problem under consideration in order to achieve convergence, and ii) selection of the optimum relaxation parameter in order to achieve maximum computational efficiency. In this respect, work by El-Gebeily et al. [17] on static coupling of BEM-FEM domains, Estorff and Hagen [18] on iterative coupling of FEM and BEM in 3D transient elasto-dynamics, and also more recent work by the authors $[19,20]$ on nonlinear coupling of soil-structure interaction using FEM, have shown that the convergence behaviour of the constant relaxation scheme, in which the relaxation parameter remains constant at all coupling iterations, is very sensitive to the problem parameters, particularly the relative stiffness of the partitioned sub- 
domains. Indeed, this leads to considerable computational inefficiency, especially for realistic large-scale nonlinear problems. The performance of interface relaxation in iterative coupling of soil-structure interaction problems may be enhanced through the use of an adaptive/dynamic relaxation [20]. In contrast with the constant relaxation scheme, in which the relaxation parameter is typically evaluated by trial and error, an adaptive relaxation scheme offers improved prospects for achieving convergence by calculating automatically an optimum relaxation parameter at each iterative stage, thus avoiding the trial and error process embedded in the constant relaxation scheme. In this regard, iterative interface relaxation approaches proposed by Funaro et al. [21] for iterative coupling of partitioned second-order elliptic problems, Wall et al. [22] and Kutler and Wall [23] focusing on iterative coupling of fluid-structure interaction problems, and Soares [24] on iterative coupling of FEM-BEM for dynamic analyses, utilize an adaptive relaxation concept in order to accelerate the coupling convergence rate.

Notwithstanding the above, it is possible to extend the existing iterative coupling methods to enhance the computational efficiency of both adaptive and constant relaxation coupling algorithms, while overcoming the problematic issues regarding the trial and error process embedded in evaluation of the constant relaxation parameter. The main contribution of this paper can be seen in this context, where it is proposed that the performance of iterative coupling methods may be effectively enhanced through the use of the condensed tangent stiffness of the structure and soil partitioned sub-domains at the interface degrees of freedom. This provides an effective first-order guide to iterative forces and displacements at the soil-structure interface, enforcing convergence to compatibility and equilibrium in successive iterations. Although the condensed tangent stiffness matrix may be readily available with some of the current nonlinear field modelling tools, as a special case, it is proposed here that a suitable and more general approximation can be obtained via constructing reduced order models of the partitioned structure and soil sub-domains. This builds on a previous approach presented by Vierendeels and co-workers [25, 26], who utilized a procedure for constructing the reduced order model of partitioned sub-domains throughout the coupling iterations for implicit coupling of fluid-structure interaction problems, though some significant modifications are proposed in this paper to provide an efficient approach for coupled modelling of nonlinear soil-structure interaction. In contrast with the traditional 
relaxation scheme, in which the relaxation parameter is typically evaluated by trial and error, the proposed approach offers improved prospects for achieving convergence and computational efficiency in large scale nonlinear problems. The evaluation of such prospects and the comparison against conventional relaxation schemes are therefore primary objectives of this paper, particularly considering the application to soilstructure interaction problems with nonlinearity in both structure and soil.

\section{Partitioning of Coupled Soil-Structure System}

Before some variant coupling algorithms are introduced, it is beneficial to illustrate the domain decomposition strategy and discretised representation of the partitioned subdomains in coupling algorithms. For a partitioned treatment of coupled soil-structure interaction problems, a common practice which allows field-specific discretisation and solution procedures would be to decompose the coupled system into two sub-domains according to their physical and material properties, namely soil and structure.

Here, it is assumed that the soil-structure interaction coupled system is partitioned into soil and structure sub-domains as shown in Fig.1, where each sub-domain is independently discretised by FEM. It should be noted that the suggested discretisation of the partitioned sub-domains is just for illustration and the proposed method is in fact applicable to different desired types of discretisation.

According to the presented partitioning strategy, the soil and structure sub-domains can be formulated independently as given below where, without loss of generality, the response of individual sub-domains is first assumed to be linear elastic:

Governing equilibrium conditions for partitioned structure sub-domain:

$\left[\begin{array}{ll}K_{11}^{T} & K_{12}^{T} \\ K_{21}^{T} & K_{22}^{T}\end{array}\right]\left\{\begin{array}{l}U_{T}^{T} \\ U_{T}^{i}\end{array}\right\}=\left\{\begin{array}{l}F_{T}^{T} \\ F_{T}^{i}\end{array}\right\}$

Governing equilibrium conditions for partitioned soil sub-domain:

$\left[\begin{array}{ll}K_{11}^{B} & K_{12}^{B} \\ K_{21}^{B} & K_{22}^{B}\end{array}\right]\left\{\begin{array}{l}U_{B}^{B} \\ U_{B}^{i}\end{array}\right\}=\left\{\begin{array}{c}F_{B}^{B} \\ F_{B}^{i}\end{array}\right\}$ 
In the above, vectors $\left\{U_{X}^{X}\right\}$ and $\left\{F_{X}^{X}\right\}$ correspond to the displacements and external forces for non-interface degrees of freedom, while $\left\{U_{X}^{i}\right\}$ and $\left\{F_{X}^{i}\right\}$ correspond to displacements and forces for interface degrees of freedom, respectively.

\section{Coupling Method}

The coupling method consists of partitioning the coupled soil-structure system, as discussed in the previous section, and of imposing some boundary conditions at the interface boundaries defined by the partitioning strategy. The resulting set of problems is solved separately, and if compatibility and equilibrium are not satisfied at the interface, the imposed boundary conditions at the interface will be iteratively corrected until convergence is ensured. This task is achieved by employing a sequential Dirichlet-Neumann $(D-N)$ iterative coupling algorithm [14]. The algorithm is formally defined by the algorithmic steps presented in Table 1, in which superscript $I$ and subscript $n$ denote the iteration number and the load/time increment number, respectively.

The partitioned structure sub-domain is considered subject to Dirichlet (displacement) boundary conditions at the interface, whereas the partitioned soil sub-domain is subjected to a Neumann (force) boundary condition at the same interface. This is purely due to the fact that in static problems only essential Dirichlet boundary conditions can be imposed on the interface of the structure sub-domain, where applying the natural Neumann boundary conditions at this interface results in singularity of the equilibrium equations for the structural sub-domain.

Here, we assume that the soil and the structure remain always in contact at the interface, while the separation and slip can be treated as an extension of this approach through the use of interface elements that may be considered to be either part of one of the subdomains, or part of the interface model.

\subsection{Relaxation Method}

The critical algorithmic stage of the procedure presented in Table 1 is the evaluation of new estimates of the interface Dirichlet data in each coupling iteration (STEP 6), which 
determines the convergence rate to compatibility at the interface, noting that interface equilibrium is already satisfied (STEP 3).

Employing a relaxation scheme is one of the most common techniques used for successive update of the boundary conditions in iterative coupling algorithms. In this method, the convergence to compatibility of the presented D-N coupling algorithm is accelerated and ensured by employing relaxation of the updated interface boundary conditions as given by Eq.(3):

$\left\{U_{T}^{i}\right\}_{n}^{I+1}=\left(1-\alpha_{I}\right)\left\{U_{T}^{i}\right\}_{n}^{I}+\alpha_{I}\left\{U_{B}^{i}\right\}_{n}^{I}$

where, $\alpha_{I} \alpha$ is a real positive relaxation parameter that can improve the convergence of the iterative scheme. In this respect, the use of a constant relaxation scheme as in Eq. (3) can enhance convergence, where it is assumed that the relaxation parameter is constant during the coupling iterations (i.e. $\alpha_{1}, \ldots, \alpha_{I}=\alpha$ ). However, the value of the relaxation parameter that ensures convergence is problem dependent. A common strategy to obtain an optimum relaxation parameter, combining convergence with the best computational efficiency, is by trial and error. Accordingly, ensuring convergence and computational efficiency in large scale nonlinear problems, where the optimum relaxation parameter is to be determined over the full range of response by trial and error, would be very difficult, if not impossible, and computationally inefficient [20].

On the other hand, in adaptive relaxation, an optimum relaxation parameter is automatically determined during each coupling iteration. In this respect the relaxation parameter is obtained, for a specific coupling iteration, from minimizing the compatibility and equilibrium defaults at the next iteration by using the compatibility and equilibrium default history of the previous iterations. The method allows for adaptive change of relaxation parameter at every iteration $(I>1)$, as given by Equation (4) [20], while for the first iterative stage $(I=1)$ the relaxation parameter can be chosen as an arbitrary value $(0<\alpha \leq 1)$ :

$$
\alpha_{I}=\frac{\alpha_{I-1}\left(\left\|\left\{\Delta_{I-1}\right\}\right\|_{2}^{2}-\left\{\Delta_{I-1}\right\}\left\{\Delta_{I}\right\}\right)}{\left\|\left\{\Delta_{I-1}\right\}-\left\{\Delta_{I}\right\}\right\|_{2}^{2}}
$$

with,

$$
\left\{\Delta_{I}\right\}=\left\{U_{B}^{i}\right\}_{n}^{I}-\left\{U_{T}^{i}\right\}_{n}^{I}
$$


This method enhances the constant relaxation scheme, as it avoids the trial and error for establishing the optimum relaxation parameter for the specific coupled system under consideration and has a higher convergence rate.

\subsection{Condensed Interface Tangent Stiffness}

Consider domain decomposition of the soil-structure interaction problem presented in Section 3 treated by D-N iterative coupling approach. The compatibility defaults at the interface of the structure and soil sub-domains for iteration number $I$ of the increment number $n$ take the form:

$\left\{\Psi_{U}\right\}_{n}^{I}=\left\{U_{B}^{i}\right\}_{n}^{I}-\left\{U_{T}^{i}\right\}_{n}^{I}$

The new estimation of the interface displacements and forces in the successive iteration $I+1$ is expressed incrementally as:

$$
\begin{aligned}
& \left\{U_{T}^{i}\right\}_{n}^{I+1}=\left\{U_{T}^{i}\right\}_{n}^{I}+\left\{\Delta U_{T}^{i}\right\}_{n}^{I} \\
& \left\{U_{B}^{i}\right\}_{n}^{I+1}=\left\{U_{B}^{i}\right\}_{n}^{I}+\left\{\Delta U_{B}^{i}\right\}_{n}^{I} \\
& \left\{F_{T}^{i}\right\}_{n}^{I+1}=\left\{F_{T}^{i}\right\}_{n}^{I}+\left\{\Delta F_{T}^{i}\right\}_{n}^{I} \\
& \left\{F_{B}^{i}\right\}_{n}^{I+1}=\left\{F_{B}^{i}\right\}_{n}^{I}+\left\{\Delta F_{B}^{i}\right\}_{n}^{I}
\end{aligned}
$$

where to a first order:

$$
\begin{aligned}
& \left\{\Delta F_{T}^{i}\right\}_{n}^{I}=\left[\mathbf{K}_{\mathbf{T}}^{\mathbf{C}}\right]\left\{\Delta U_{T}^{i}\right\}_{n}^{I} \\
& \left\{\Delta F_{B}^{i}\right\}_{n}^{I}=\left[\mathbf{K}_{\mathbf{B}}^{\mathbf{C}}\right]\left\{\Delta U_{B}^{i}\right\}_{n}^{I}
\end{aligned}
$$

in which $\left[\mathbf{K}_{\mathbf{T}}^{\mathbf{C}}\right]$ and $\left[\mathbf{K}_{\mathbf{B}}^{\mathbf{C}}\right]$ are the condensed tangent stiffness matrices at the interface of the structure and soil sub-domains.

It can be shown that in order to achieve convergence to compatibility at iteration $I+1$ at the interface of the partitioned soil structure system, the value of $\left\{\Delta U_{T}^{i}\right\}_{n}^{I}$ should take the following form:

$$
\left\{\Delta U_{T}^{i}\right\}_{n}^{I}=([\mathbf{I}]+[\lambda])^{-1}\left\{\Psi_{U}\right\}_{n}^{I}
$$


where $[\mathbf{I}]$ is the identity matrix and $[\lambda]=\left[\mathbf{K}_{\mathbf{B}}^{\mathbf{C}}\right]^{-1}\left[\mathbf{K}_{\mathbf{T}}^{\mathbf{C}}\right]$.

Therefore, renewal of the boundary conditions can be expressed as:

$$
\left\{U_{T}^{i}\right\}_{n}^{I+1}=\left\{U_{T}^{i}\right\}_{n}^{I}+([\mathbf{I}]+[\lambda])^{-1}\left\{\Psi_{U}\right\}_{n}^{I}
$$

The above equation can be used in successive update of boundary conditions of the presented sequential D-N iterative coupling algorithms (STEP 6), and can be rewritten in an alternative form:

$\left\{U_{T}^{i}\right\}_{n}^{I+1}=([\mathbf{I}]-[\boldsymbol{\beta}])\left\{U_{T}^{i}\right\}_{n}^{I}+[\boldsymbol{\beta}]\left\{U_{B}^{i}\right\}_{n}^{I}$

where $[\boldsymbol{\beta}]=([\mathbf{I}]+[\boldsymbol{\lambda}])^{-1}$

It can be immediately noted that Eq. (14) resembles the interface relaxation scheme presented in Eq. (3). However, $[\boldsymbol{\beta}]=([\mathbf{I}]+[\lambda])^{-1}$ is a fully populated relaxation matrix, as opposed to the scalar relaxation matrix of Eq. (3) that guarantees convergence to equilibrium and compatibility at the interface of the partitioned sub-domains while holding an optimum convergence rate. The proposed approach brings the numerical performance of iterative coupling approach close to the monolithic treatment, whilst maintaining the practical and computational benefits of the partitioned treatment. Moreover, in contrast with the constant relaxation coupling algorithms, this method does not require the definition of certain parameters by a process of trial and error for each case under consideration. Although the proposed approach involves more interface-related computations than the relaxation approach, these computations are relatively minor in comparison with those undertaken in the soil and structure models, and therefore superior overall computational efficiency is envisaged due to the enhanced convergence of iterative coupling.

The convergence of the presented sequential D-N algorithms can be established for a linear case by following the algorithmic steps presented in Table 1, while assuming the iteratively updated boundary conditions in Step 6 to have the following general form:

$\left\{U_{T}^{i}\right\}_{n}^{I+1}=([\mathbf{I}]-[\boldsymbol{X}])\left\{U_{T}^{i}\right\}_{n}^{I}+[\boldsymbol{X}]\left\{U_{B}^{i}\right\}_{n}^{I}$

where $[\boldsymbol{X}]$ is an unknown matrix which is to be determined to ensure convergence of the iterative coupling algorithm. 
Substituting Eqs. (1) and (2) for $K$ consecutive iterations, while using Eq. (17) for renewal of the interface Dirichlet data, the compatibility default after $K$ iterations at the interface of the decomposed soil-structure system, can be obtained by the following difference equation:

$\left\{U_{B}^{i}\right\}^{K}-\left\{U_{T}^{i}\right\}^{K}=([\mathbf{I}]-[\mathbf{X}]([\mathbf{I}]+[\lambda]))^{K}\left(\left\{U_{B}^{i}\right\}^{1}-\left\{U_{T}^{i}\right\}^{1}\right)$

with

$[\lambda]=\left[\mathbf{K}_{\mathbf{B}}^{\mathbf{C}}\right]^{-1}\left[\mathbf{K}_{\mathbf{T}}^{\mathbf{C}}\right]$

$\left[\mathbf{K}_{\mathbf{T}}^{\mathbf{C}}\right]=\left(\left[K_{22}^{T}\right]-\left[K_{21}^{T}\right]\left[K_{11}^{T}\right]^{-1}\left[K_{12}^{T}\right]\right):$ Condensed stiffness matrix of the structure subdomain corresponding to the interface degrees of freedom.

$\left[\mathbf{K}_{\mathbf{B}}^{\mathbf{C}}\right]=\left(\left[K_{22}^{B}\right]-\left[K_{21}^{B}\right]\left[K_{11}^{B}\right]^{-1}\left[K_{12}^{B}\right]\right)$ : Condensed stiffness matrix of the soil subdomain corresponding to the interface degrees of freedom.

For the successive iteration process of Eq. (18) to converge for any initial value, $\left\{U_{T}^{i}\right\}_{n}^{I}=\{\bar{U}\}$, it can be clearly shown that $[\boldsymbol{X}]$ should take the form of:

$[\mathbf{X}]=[\boldsymbol{\beta}]=([\mathbf{I}]+[\boldsymbol{\lambda}])^{-1}$

in which case convergence to compatibility occurs for linear problems immediately at the second iteration $(K=2)$. Of course, this immediate convergence does not normally occur for nonlinear problems, though use of Eq. (15) to update the interface Dirichlet boundary conditions ensures a superior convergence rate to the relaxation approach expressed by Eq.(3).

\section{Approximation of the Condensed Tangent Stiffness}

While the condensed tangent stiffness matrix can be determined with current nonlinear field modelling tools, it is proposed here that it may be reasonably approximated by constructing reduced order models $[25,26]$ of the structure and soil sub-domains. The benefit of such an approach is that it does not require the explicit assembly of the stiffness matrices, thus providing a potentially efficient coupling technique.

The interface condensed tangent stiffness matrices of the partitioned soil and structure sub-domains can be approximated at each coupling iteration stage $(I \geq 2)$ of a particular 
time/load increment by constructing the following reduced order model of the partitioned sub-domains. Considering the partitioned structure sub-domain, the following displacement mode matrix can be constructed for the structure partitioned sub-domain at coupling iteration $I \geq 2$ :

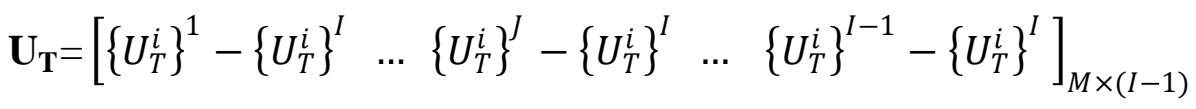

where $\mathrm{M}$ corresponds to the number of coupled degrees of freedom at soil structure interface and $J=1, \ldots, I-1$.

Similarly, the variation of the interface forces corresponding to $\mathbf{U}_{\mathbf{T}}$ can be constructed as:

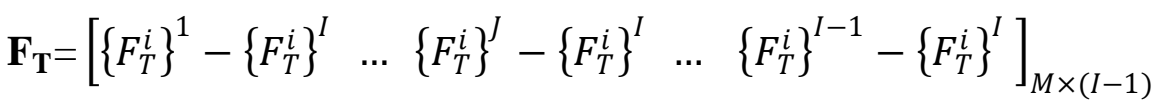

Considering Eq. (7), $\left\{\Delta U_{T}^{i}\right\}_{n}^{I}$ can be projected as a linear combination of displacement modes given by Eq. (21):

$\left\{\Delta U_{T}^{i}\right\}_{n}^{I} \approx \mathbf{U}_{\mathbf{T}} \cdot \boldsymbol{\delta}$

where $\boldsymbol{\delta}=\left\langle\begin{array}{lllll}\delta_{1} & \ldots & \delta_{J} & \ldots & \delta_{I-1}\end{array}\right\rangle^{\mathrm{T}}$

Thus the variation of the interface forces can also be approximated in a similar manner:

$\left\{\Delta F_{T}^{i}\right\}_{n}^{I} \approx \mathbf{F}_{\mathbf{T}} \cdot \boldsymbol{\delta}$

The coefficients $\boldsymbol{\delta}$ which provide the minimum error in approximating any $\left\{\Delta U_{T}^{i}\right\}_{n}^{I}$ according to Eq.(23) can be obtained as:

$\boldsymbol{\delta}=\left(\left[\mathbf{U}_{\mathbf{T}}\right]^{T}\left[\mathbf{U}_{\mathbf{T}}\right]\right)^{-\mathbf{1}}\left[\mathbf{U}_{\mathbf{T}}\right]^{T}\left\{\Delta U_{T}^{i}\right\}_{n}^{I}$

Now, the reduced order model of the partitioned structure sub-domain can be determined from combining Eqs. (25) and (26):

$\left\{\Delta F_{T}^{i}\right\}_{n}^{I}=\left[\mathbf{F}_{\mathbf{T}}\right]\left(\left[\mathbf{U}_{\mathbf{T}}\right]^{T}\left[\mathbf{U}_{\mathbf{T}}\right]\right)^{-\mathbf{1}}\left[\mathbf{U}_{\mathbf{T}}\right]^{T}\left\{\Delta U_{T}^{i}\right\}_{n}^{I}$ 
Comparing the reduced order model of the structure given by Eq. (27) with Eq. (11), it is evident that the condensed tangent stiffness matrix of the partitioned structure subdomain can be approximated as:

$\left[\mathbf{K}_{\mathbf{T}}^{\mathbf{C}}\right]=\left[\mathbf{F}_{\mathbf{T}}\right]\left(\left[\mathbf{U}_{\mathbf{T}}\right]^{T}\left[\mathbf{U}_{\mathbf{T}}\right]\right)^{-\mathbf{1}}\left[\mathbf{U}_{\mathbf{T}}\right]^{T}$

In a similar manner, the inverse condensed tangent stiffness matrix of the partitioned soil sub-domain can be obtained as:

$\left[\mathbf{K}_{\mathbf{B}}^{\mathbf{C}}\right]^{-1}=\left[\mathbf{U}_{\mathbf{B}}\right]\left(\left[\mathbf{F}_{\mathbf{B}}\right]^{T}\left[\mathbf{F}_{\mathbf{B}}\right]\right)^{-\mathbf{1}}\left[\mathbf{F}_{\mathbf{B}}\right]^{T}$

where $\left[\mathbf{U}_{\mathbf{B}}\right]$ and $\left[\mathbf{F}_{\mathbf{B}}\right]$ are obtained in a similar way to $\left[\mathbf{U}_{\mathbf{T}}\right]$ and $\left[\mathbf{F}_{\mathbf{T}}\right]$ in Eqs. (21) and (22).

The abovementioned approximations of the condensed stiffness matrices at the interface, as given by Eqs. (28) and (29), can be used in successive updates of the boundary conditions of Eq.(14). The coupling procedure of soil-structure interaction problems using the presented sequential D-N coupling method is also illustrated in Table 1. It is worth noting that, in the proposed scheme by Vierendeels et al. [26] for coupling fluid-structure interaction problems, the prescribed Neumann data are calculated as a result of solving the reduced order models of the partitioned subdomains in consecutive iterations, (except for the first two iterations, where equilibrium is applied). Here, the reduced order models are solved only once to obtain the prescribed Dirichlet data to structure sub-domain (STEP 6), while the prescribed Neumann data to the soil sub-domain is obtained by enforcing equilibrium at all iterations (STEP 3).

\section{Simulation Environment for Nonlinear Soil-Structure Interaction}

A coupling simulation environment, utilising discipline-oriented solvers for nonlinear structural and geotechnical analysis, has been developed to simulate the coupled modelling of nonlinear soil-structure interaction via the partitioned approach. More specifically, the developed environment is based on the coupling of the two disciplineoriented FEM codes ADAPTIC [27] and ICFEP [28], which have been developed at Imperial College London for advanced nonlinear structural and geotechnical analysis, respectively. Importantly, however, while the developed methods are applied to the 
coupling of ADAPTIC and ICFEP, these methods are general in nature and applicable to the coupling of other existing nonlinear soil and structural software.

The coupling of ADAPTIC and ICFEP is carried out using a computer program called INTERFACE, written in FORTRAN 95, which utilises a sequential Dirichlet-Neumann type of iterative coupling algorithm. Within the overall environment, ADAPTIC and ICFEP run on separate processors as independent black box solvers, residing on LINUX and SUN platforms respectively, where the task of communication and synchronization between the two individual codes is achieved via INTERFACE. In this respect, the interface program manages the retrieval, manipulation and passing of the necessary data between the two field programs during coupled analysis (Fig. 2).

The developed simulation environment is based on client-server software architecture, communicating over a computer network, in which the client software, here the INTERFACE program, can send data requests to the connected servers, ADAPTIC and ICFEP, which in turn accept these requests, process them, and return the requested information to the client.

At the start of coupled modelling, the user specifies in ADAPTIC and ICFEP the input files corresponding to structure and soil partitioned sub-domains. These contain the typical relevant information such as geometric modelling, material modelling, analysis type, etc. In addition to these, an input file for the INTERFACE program is specified which defines the coupling region for both the structure and soil partitioned models. During analysis, communication between the INTERFACE, ADAPTIC and ICFEP programs is undertaken via a direct access file, as illustrated in Fig. 3. A flowchart of the coupled analysis procedure is provided in Fig. 4, which mirrors the sequential Dirichlet-Neumann procedure presented in Table 1.

The developed simulation environment is used in the following section to demonstrate the relative performance characteristics and merits of the presented algorithms, and to illustrate the benefits of iterative coupling based on the condensed stiffness matrix at the coupling interface.

\section{Example Applications}

In this section two examples of soil-structure coupling are presented, utilizing both the relaxation scheme and the reduced order method. 
The first example is a simple linear soil-structure interaction problem as presented in Fig. 5, which is aimed at verifying the developed coupled simulation environment against the monolithic treatment. The problem consists of linear static plane strain analysis of a concrete cantilever wall resting on a flexible soil, loaded at the top with a horizontal force. In partitioned analysis of the problem, both adaptive relaxation and the reduced order method show similar reasonable convergence characteristics and converge within 3 coupling iterations to a prescribed tolerance of $10^{-4} \mathrm{~m}$. With the same problem also modelled monolithically, the obtained results from both monolithic and partitioned analysis are in excellent agreement, as illustrated in Fig. 6. This figure shows the horizontal displacement of the beam obtained for the non-interactive case (rigid base) and for the interactive case by both the partitioned and monolithic approaches. This further demonstrates that by enforcing convergence to equilibrium and compatibility in the sequential D-N iterative coupling algorithm, a strong coupling of the partitioned sub-domains can be achieved at the interface.

Fig. 7 represents the recorded CPU-time for analysing the above example with both the monolithic and partitioned approaches. Comparing the computational efficiency of the monolithic approach with that of the partitioned approach via the sequential DirichletNeumann algorithm, it is clear that the monolithic treatment, if available, would be more efficient than the sequential iterative coupling approach, since the former does not require coupling iterations. As evident from Fig.7, the CPU-time of the partitioned soil and structure partitioned sub-domains for 3 coupling iterations are respectively 2.3 and 2.5 times higher than the monolithic approach. It is also clear that the CPU-time demand of the INTERFACE program (0.04 s) is insignificant compared to that of partitioned subdomains.

The second example represents a typical urban situation, where ground works can often induce significant movements and damage to the nearby structures. The example considers a steel frame resting on a soil subjected to ground excavation, where nonlinear elasto-plastic constitutive behaviour of the soil as well as geometric and material nonlinearity of the structure are taken into account. Fig. 8 depicts the problem under consideration, where the left hand side boundary of the problem is assumed to be consistent with an axis of symmetry.

The plan view of the analysed building frame is shown in Fig. 9. The building is designed for office purposes and is assumed to be loaded equally on each floor with a 
total gravity load equal to $5 \mathrm{kN} / \mathrm{m}^{2}$. The span length and the storey height of the steel frame are $6 \mathrm{~m}$ and $3.5 \mathrm{~m}$, respectively, except for the ground floor where the storey height is $4 \mathrm{~m}$, as shown in Fig. 10 .

The soil-structure interaction analysis is carried out assuming plane strain conditions in the soil using an effective out-of-plane width of $1 \mathrm{~m}$, where the developed domain decomposition approach is employed utilising ADAPTIC and ICFEP. The main objective of this study is to establish the applicability and efficiency of the presented coupling algorithm using the reduced order method, highlighting its merits compared to relaxation scheme.

According to the partitioned treatment, the considered soil-structure system is partitioned physically into two sub-domains, soil and structure, where each sub-domain is discretised separately according to its characteristics as shown in Table 2.

The frame structure is modelled with ADAPTIC using cubic elasto-plastic beamcolumn elements [29], which enable the modelling of geometric and material nonlinearity. The frame is discretised using 10 elements per member for both columns and beams, and the material behaviour is assumed to be bilinear elasto-plastic with kinematic strain hardening. The footings are discretised using 4 elements per member.

The soil sub-domain is modelled with ICFEP using an elasto-plastic Mohr-Coulomb constitutive model, with parameters chosen to represent the behaviour of London clay (Table 2). The nonlinear solution procedure employed for analysing the soil sub-domain is based on Modified Newton-Raphson technique, with an error controlled sub-stepping stress point algorithm. The soil continuum is discretised using 8-noded isoparametric quadrilateral elements, while the excavation in the soil and the loads on the structure are applied in six increments. The interface degrees of freedom are assumed to be at nodes that belong to both the footings and soil underneath. The total number of degrees of freedom is 30 for this case.

To assess the merits of presented coupling schemes, both relaxation and reduced order methods are employed in modelling this soil structure interaction problem. For the relaxation approach, the considered problem is analysed using different constant relaxation parameters $(\alpha \in] 0,1])$. Fig.11 shows the total number of coupling iterations required for various constant relaxation parameters and the applicable range of relaxation parameters ensuring convergence to compatibility over the full range of 
response consisting of six increments. A tolerance of $\varepsilon=10^{-4} \mathrm{~m}$ was set for the compatibility error of each coupled degree of freedom at the interface, with the convergence criterion taken as follows:

Compatibility Error $=\left\|\left\{U_{T}^{i}\right\}^{I}-\left\{U_{B}^{i}\right\}^{I}\right\| \leq \sqrt{M} \times$ Tolerance

where M corresponds to the number of coupled degrees of freedom at the soil structure interface.

The results confirm that the convergence behaviour of the iterative scheme using constant relaxation is very sensitive to the chosen relaxation parameter, rendering its selection a very difficult task, as evidenced by the significant increase in number of iterations between $\alpha=0.5$ with 80 coupling iterations, $\alpha=0.52$ with 121 coupling iterations and $\alpha=0.55$ with 260 coupling iterations.

Table 3 presents the range of constant relaxation parameters which guarantee convergence, the optimum relaxation parameter and the number of coupling iterations required for convergence to the prescribed tolerance for the reduced order method, constant relaxation and adaptive relaxation methods. The convergence rates of the three approaches in the first, third and last load increments are illustrated in Figs. 12, 13 and 14 respectively. This demonstrates that the reduced order method achieves a faster convergence rate than the relaxation scheme, whether optimal or not.

Fig. 15 shows the number of coupling iterations required in each increment for different coupling schemes, which demonstrates that the optimum relaxation parameter should be determined over the full range of response in nonlinear problems. This is evidenced by considering the behaviour of coupling with $\alpha=0.5$, where a relatively good convergence rate is observed in the first two load increments (better than the optimum relaxation with $\alpha=0.45$ ), but as the coupled system presents more nonlinearity the convergence rate decreases significantly. Indeed, as the results clearly indicate, the reduced order method is far superior to the constant relaxation scheme, since not only it enhances the convergence rate of the coupling method, but also it does not require the trial and error process of determining an adequate relaxation parameter. It is also clear that the reduced order method has a higher convergence rate compared to adaptive relaxation. 
According to Eqs. (20) and (28), the reduced order method converges when a good approximation of the condensed tangent stiffness is achieved. Considering Fig. 14, it can be seen that in the last increment this is achieved through ten cycles of displacement/force history data, while in the first load increment (Fig. 12) this is achieved via three cycles. In fact, the more displacement/force history data (i.e. the more coupling iterations) the better the approximation of the tangent stiffness matrix. It is however worth observing that the required number of coupling iterations for convergence using the reduced order method is still relatively large in comparison with what would be necessary in a typical monolithic treatment. Indeed, if a better approximation of the condensed tangent stiffness matrix could be achieved, the coupling algorithms will become even more robust, thus highlighting the potential for further enhancement.

Notwithstanding, the benefits of the developed simulation environment in the practical assessment of nonlinear soil-structure interaction problems can be demonstrated by considering the results of this case study. The frame structure with its applied loads transmits the loading to the soil, which in turn deforms due to cumulative action of these loads and the excavation. As a consequence, the soil deformation beneath the structure transmits back additional deformations and corresponding forces to the structure. This interactive process is continued until the whole coupled system reaches a compatible equilibrium state. In the following, the deformation and stress states of the coupled problem at the end of excavation and load application are briefly described.

The vertical deformation profile of the soil surface with respect to the distance from the excavation edge obtained from coupled analysis is given in Fig.16, where the three troughs correspond to the locations of the three footings showing their vertical settlement. Clearly, the generated level of vertical settlement, of the order of $15 \mathrm{~cm}$, requires the structural analysis model to account for geometric nonlinearity. On the other hand, the horizontal movement of the excavation wall is presented in Fig. 17, where the maximum horizontal displacement is more than $22 \mathrm{~cm}$.

A vector plot of displacements in the soil sub-domain in the vicinity of the structure and excavation is also shown in Fig. 18. While the absolute magnitudes of these vectors are not important, it is their relative magnitude that shows the mechanism of ground deformation. 
Fig. 19 shows contours of stress level after the full excavation to $6 \mathrm{~m}$ depth is achieved under the applied load on the structure. The stress level is the ratio, at the same mean effective stress, of the current deviatoric stress to the deviatoric stress at failure. It therefore varies from 0 to 1 , where 1 indicates full plasticity and failure. It is evident from Fig. 19 that the applied loading conditions have mobilised an extensive plastic zone underneath the building. This zone is, however, smaller and shallower under the right hand side footing, which is in agreement with the previous figures that show most of the deformation and load concentration nearer the excavation.

The deformed shape and the bending moment contours of the partitioned structure subdomain are shown in Fig. 20. The presented frame is also analysed using field elimination approach, where linear springs with an approximated stiffness of 10,000 $\mathrm{kPa}$ are used to represent the soil domain without considering the effect of nearby excavation. The deformed shape and bending moment variation in the frame for the field elimination case are given in Fig. 21. It can be clearly observed that the bending moment of the structural elements in the fully coupled analysis (Fig. 20) is significantly higher than that of field elimination analysis, where significant plastic deformation is observed in the fully coupled scenario. The comparison of the bending moment values for six selected regions, A, B, C, D, E and F as shown in Fig. 20, is presented for both fully coupled and field elimination analysis in Table 4.

It is evident from the deformed shape of the structure in fully coupled interaction analysis and also the vectors underneath each of the three footings in Fig.18 that they experience rigid tilting (indicated previously in Fig. 16) and significant vertical settlements. However, the footing nearest to the excavation has the smallest tilting, as its deformation is also dominated by the horizontal movement towards the unsupported excavation.

\section{Conclusion}

This paper presents a domain decomposition method for nonlinear analysis of soilstructure interaction problems, where particular emphasis was given to iterative coupling methods via a reduced order method. The overall domain is divided into physical partitions consisting of soil and structure sub-domains, and coupling of the separately modelled sub-domains is undertaken via sequential iterative DirichletNeumann sub-structuring method. An important feature of the proposed approach is the 
utilisation of the condensed tangent stiffness matrices at the interface of partitioned soil and structure sub-domains to ensure and accelerate convergence to compatibility in successive update of the boundary conditions. In this respect, the condensed tangent stiffness matrix is approximated via reduced order models of partitioned domains throughout the coupling iterations.

It is shown that the reduced order method provides good convergence characteristics. Moreover, its superiority not only in terms of convergence rate but also avoiding the trial and error process embedded in the selection of an optimal - even adequate relaxation parameter is established.

The computational demand of the partitioned approach is also compared to that of the monolithic treatment, where it is shown that the presented partitioned approach is typically less efficient than the monolithic treatment. However, with the lack of suitable monolithic tools, the partitioned approach provides a powerful framework for modelling nonlinear soil-structure interaction problems through the coupling of existing fieldspecific codes developed in isolation for nonlinear structural and geotechnical analysis. The applicability of the presented technique is demonstrated for nonlinear soil-structure interaction analysis via a case study investigating the interactive behaviour of a plane frame and a supporting soil system subject to ground excavation. In this context, the proposed method is shown to offer great potential towards providing an integrated interdisciplinary computational approach which combines the advanced features of both structural and geotechnical modelling for a variety of challenging problems in the field of nonlinear soil-structure interaction.

Finally, it is worth noting that the computational efficiency of the presented partitioned approach may be further enhanced on two fronts. Firstly, faster convergence of iterative coupling may be achieved by constructing a more accurate approximation of the condensed stiffness matrix. Secondly, parallel coupling algorithms could also improve computational efficiency provided that the computational demands of the partitioned soil and structure sub-domains are of the same order. Unlike the presented sequential coupling treatment, in parallel coupling the partitioned sub-domains are solved concurrently at each coupling iteration [30,31]. Such enhancements and their convergence characteristics are currently being investigated by the authors, where the main emphasis is on the development of coupling algorithms for nonlinear soil-structure interaction utilising existing structural and geotechnical nonlinear analysis codes. 


\section{References}

[1] Felippa CA, Park KC, Farhat C. Partitioned analysis of coupled mechanical systems. Computer Methods in Applied Mechanics and Engineering 2001; 190:3247-3270.

[2] Rugonyi S, Bathe KJ. On the finite element analysis of fluid flows fully coupled with structural interactions. Computer Modeling in Engineering \& Sciences 2001; 2:195-212.

[3] Lai CH. Diakoptics, domain decomposition and parallel computing. The Computer Journal 1994; 37(10).

[4] Navon IM, Cai Y. Domain decomposition and parallel processing of a finite element model of the shallow water equations. Computer Methods in Applied mechanics and Engineering 1993; 106:179-212.

[5] Kamiya N, Iwase H, Kita E. Parallel implementation of boundary element method with domain decomposition. Engineering Analysis with Boundary elements 1996; 18:209-216.

[6] Park KC, Felippa CA, Deruntz JA. Stabilization of staggered solution procedures for fluid-structure interaction analysis. Proceedings of the Winter Annual Meeting, Atlanta, Ga ; United States; 27 Nov.-2 Dec. 1977,pp95-124, 1997.

[7] Farhat C, Lesoinne M. Two efficient staggered algorithms for serial and parallel solution of three-dimensional nonlinear transient aeroelastic problems. Computer Methods in Applied Mechanics and Engineering 2000; 182:499-515.

[8] Zienkiewicz OC, Taylor RL. Finite Element Method Solid and Fluid Mechanics: Dynamics and Nonlinearity, Vol. 2, New York; McGraw-Hill, 1991.

[9] Quarteroni A, Valli A. Domain Decomposition Methods for Partial Differential Equations, Oxford; Clarendon press, 1999.

[10] Rizos DC, Wang Z. Coupled BEM-FEM solutions for direct time domain soilstructure interaction analysis. Engineering Analysis with Boundary Elements $2002 ; 26,877-888$.

[11] O'Brien J, Rizos DC. A 3D BEM-FEM methodology for simulation of high speed train induced vibrations. Soil Dynamics and Earthquake Engineering 2005; 25, 289-301.

[12] Marini LD, Quarteroni A. A relaxation procedure for domain decomposition methods using finite elements. Numerische Mathematik 1989; 55, 575-598.

[13] ElleithyWM, Al-GahtaniHJ, El-Gebeily M. Iterative coupling of BE and FE methods in elastostatics. Engineering Analysis with Boundary Elements 2001; 25, 685-695. 
[14] Elleithy WM, Tanaka M. Interface relaxation algorithms for BEM-BEM coupling and FEM-BEM coupling. Computer Methods in Applied Mechanics and Engineering; 2003, 192, 2977-2992.

[15] Hagen C, Estorff O. Transient dynamic investigation of 3D dam-reservoir-soil problems using an iterative coupling approach. International Conference on Computational Methods for Coupled Problems in Science and Engineering (Coupled Problems 2005), Santorini, 2005.

[16] Francois S, Masoumi HR, Degrande G. An iterative coupled boundary-finite element method for the dynamic response of structures. III European Conference on Computational Mechanics Solids, Structures and Coupled Problems in Engineering, C.A. Mota Soares et al. (eds.), Lisbon, Portugal, 5-8 June 2006.

[17] El-Gebeily M, Elleithy WM,Al-Gahtani HJ. Convergence of the domain decomposition finite element-boundary element coupling methods. Computer Methods in Applied Mechanics and Engineering 2002; 191, 4851-4867.

[18] Estorff O, Hagen C. Iterative coupling of FEM and BEM in 3D transient elastodynamics. Engineering Analysis with Boundary Elements 2005; 29, 775 787.

[19] Jahromi HZ, Izzuddin BA, Zdravkovic L. Coupling methods for modelling nonlinear soil-structure interaction, The 15th UK Conference of the Association of Computational Mechanics in Engineering, In: B.H.V. Topping, editor, Paper no. 78, Stirlingshire, Scotland, Civil-Comp Press, 2007.

[20] Zolghadr Jahromi H, Izzuddin BA, Zdravkovic L. Partitioned analysis of nonlinear soil-structure interaction using iterative coupling, Interaction and Multiscale Mechanics 2007; 1, 1, 33-51.

[21] Funaro D, Quarteroni A, Zanolli, P. An iterative procedure with interface relaxation for domain decomposition methods. SIAM Journal on Numerical Analysis 1998; 25, 6, 1213-1236.

[22] Wall WA, Genkinger S, Ramm E. A strong coupling partitioned approach for fluid structure interaction with free surfaces. Computers and Fluids 2007; 36, 169183.

[23] Kuttler U, Wall WA. Fixed-point fluid-structure interaction solvers with dynamic relaxation. Computational Mechanics 2008; 43, 61-72.

[24] Soares D Jr. An optimised FEM-BEM time-domain iterative coupling algorithm for dynamic analyses, Computers \& Structures 2008; 86, 1839-1844.

[25] Vierendeels J, Lanoye L, Degroote J, Verdonck P. Implicit coupling of partitioned fluid-structure interaction problems with reduced order models. Computers and Structures 2007; 85:970-976. 
[26] Vierendeels J. Implicit coupling of partitioned fluid-structure interaction solvers using reduced-order models. In: Bungartz, Schäfer, editors. Fluid-structure interaction. Modelling, simulation, optimisation. Series: lecture notes in computational science and engineering, 53. Springer; 2006, ISBN 3-540-34595-7. p. $1-18$.

[27] Izzuddin BA. Nonlinear Dynamic Analysis of Framed Structures, PhD Thesis, Dept. of Civil Engineering, Imperial College, University of London, 1991.

[28] Potts DM, Zdravkovic L. Finite Element Analysis in Geotechnical Engineering: Theory. London; Thomas Telford, 1999.

[29] Izzuddin BA, Elnashai AS. Adaptive Space Frame Analysis .2. A Distributed Plasticity Approach. Proceedings of the Institution of Civil Engineers-Structures and Buildings 1993; 99, 317-326.

[30] Papadrakakis M, Bitzarakis S. Domain decomposition PCG methods for serial and parallel processing. Advances in Engineering Software; 1996, 25, 291-307.

[31] Fragakis Y, Papadrakakis M. The mosaic of high performance domain decomposition methods for structural mechanics: Formulation, interrelation and numerical efficiency of primal and dual methods. Computer methods in applied mechanics and engineering 2003; 192, 3799-3830. 
Table 1

Sequential Dirichlet-Neumann iterative coupling of soil-structure interaction

For $n=1,2, \ldots$ (number of load/time increments)

For $I=1,2, \ldots$ (number of iterations)

STEP 1: At the start of each increment, the structure domain is loaded by the external forces $\left\{F_{T}^{T}\right\}_{n}$, while the displacements at the interface nodes, $\left\{U_{T}^{i}\right\}_{n}^{I}$, are prescribed in accordance with the initial conditions: $\left\{U_{T}^{i}\right\}_{n}^{I}=\{\bar{U}\}$

STEP 2: The structural solver computes the response of the structure for $\left\{U_{T}^{T}\right\}_{n}^{I}$ and $\left\{F_{T}^{i}\right\}_{n}^{I}$.

STEP 3: The corresponding interface forces at the soil domain can be calculated by applying equilibrium: $\left\{F_{T}^{i}\right\}_{n}^{I}+\left\{F_{B}^{i}\right\}_{n}^{I}=0$

STEP 4: Based on these forces, $\left\{F_{B}^{i}\right\}_{n}^{I}$, and the external loading applied to the soil domain, $\left\{F_{B}^{B}\right\}_{n}$, the soil solver computes the response of the soil domain for $\left\{U_{B}^{i}\right\}_{n}^{I}$ and $\left\{U_{B}^{B}\right\}_{n}^{I}$.

By comparing $\left\{U_{B}^{i}\right\}_{n}^{I}$ and $\left\{U_{T}^{i}\right\}_{n}^{I}$, the following two scenarios can occur:

STEP 5: If convergence to compatibility at the interface of partitioned domains has been achieved the solution proceeds to the next time/load increment.

If $\left(\left\|\left\{\Psi_{U}\right\}_{n}^{I}\right\|=\left\|\left\{U_{T}^{i}\right\}^{I}-\left\{U_{B}^{i}\right\}^{I}\right\|\right) \leq($ Convergence Criterion), then $n=n+1$ and go to STEP 1

STEP 6: If convergence to compatibility has not been achieved, the new estimation of the displacements will be applied to the structure domain and the iteration will continue until convergence to compatibility is achieved.

(i) Relaxation:

$\left\{U_{T}^{i}\right\}_{n}^{I+1}=\left(1-\alpha_{I}\right)\left\{U_{T}^{i}\right\}_{n}^{I}+\alpha_{I}\left\{U_{B}^{i}\right\}_{n}^{I}, I=I+1$ and go to STEP 2

(ii) Reduced order method:

If $I<2$ :

Apply interface relaxation (simply using $\alpha=1$ ):

$\left\{U_{T}^{i}\right\}_{n}^{I+1}=(1-\alpha)\left\{U_{T}^{i}\right\}_{n}^{I}+\alpha\left\{U_{B}^{i}\right\}_{n}^{I}, I=I+1$ and go to STEP 2

If $I \geq 2$ :

Construct the condensed tangent stiffness matrices of the soil and structure partitioned sub-domains for iterative update of boundary conditions:

$$
\left\{U_{T}^{i}\right\}_{n}^{I+1}=\left\{U_{T}^{i}\right\}_{n}^{I}+([\mathbf{I}]+[\lambda])^{-1}\left\{\Psi_{U}\right\}_{n}^{I}, I=I+1 \text { and go to STEP } 2
$$


Table 2

Geometric and material properties of the partitioned soil-structure system

\begin{tabular}{ll}
\hline \multicolumn{1}{c}{ Structure Sub-domain } & \multicolumn{1}{c}{ Material Properties } \\
\hline $\begin{array}{l}\text { All beams and columns } \\
\text { (steel) }\end{array}$ & Steel Grade $=\mathrm{S} 355$ \\
& Elastic Modules $=210 \mathrm{GPa}$ \\
& Strength $=355 \mathrm{MPa}$ \\
& Bilinear elasto-plastic with strain Hardening Factor $=1 \%$ \\
Foundation Beam & Elastic Modulus $=30 \mathrm{GPa}$ \\
(concrete) & Linear material \\
\hline \multicolumn{1}{c}{ Soil Sub-domain } & \multicolumn{1}{c}{ Material Properties } \\
\hline Soil and excavation & Angle of Shear resistance $\left(\Phi^{\prime}\right)=22^{\circ}$ \\
& Dilation angle $\left(v^{\prime}\right)=11^{\circ}$ \\
& Effective out of plane depth $=1 \mathrm{~m}$ \\
& Cohesion $=20 \mathrm{kPa}$ \\
& Young's modulus varies linearly with depth from 10000 \\
& kPa at the ground surface $(\mathrm{d} E / \mathrm{d} Z=5000 \mathrm{kPa} / \mathrm{m})$ \\
& Excavation width $=20 \mathrm{~m} / \mathrm{depth}=6 \mathrm{~m}-$ Distance from the \\
& structure $=2 \mathrm{~m}$ \\
& Elasto-plastic Mohr-Coulomb constitutive model \\
&
\end{tabular}


Table 3

Convergence characteristics of relaxation schemes and reduced order method

\begin{tabular}{ccccc}
\hline $\begin{array}{c}\text { Relaxation } \\
\text { Range }\end{array}$ & $\begin{array}{c}\text { Optimum } \\
\text { relaxation }\end{array}$ & $\begin{array}{c}\text { Total number } \\
\text { of iterations } \\
\text { with } \\
\text { optimum } \\
\text { relaxation }\end{array}$ & $\begin{array}{c}\text { Total number } \\
\text { of iterations } \\
\text { with } \\
\text { Adaptive } \\
\text { Relaxation }\end{array}$ & $\begin{array}{c}\text { Total number } \\
\text { of iterations } \\
\text { with } \\
\text { reduced } \\
\text { order method }\end{array}$ \\
\hline$[0.1-0.6[$ & 0.45 & 58 & 48 & 43 \\
\hline
\end{tabular}


Table 4

Comparison of maximum bending moment in fully coupled and field elimination analysis

\begin{tabular}{ccc}
\hline Region & $\begin{array}{c}\mathrm{M}_{\mathrm{z}}(\mathrm{kN}-\mathrm{m}) \\
\text { Fully Coupled }\end{array}$ & $\begin{array}{c}\mathrm{M}_{\mathrm{z}}(\mathrm{kN}-\mathrm{m}) \\
\text { Field Elimination }\end{array}$ \\
\hline A & 201 & 43 \\
B & 70 & 27 \\
$\mathrm{C}$ & 170 & 41 \\
$\mathrm{D}$ & 63 & 27 \\
E & 61 & 27 \\
F & 58 & 51 \\
\hline
\end{tabular}


Figure 1: Partitioned treatment of coupled soil structure interaction

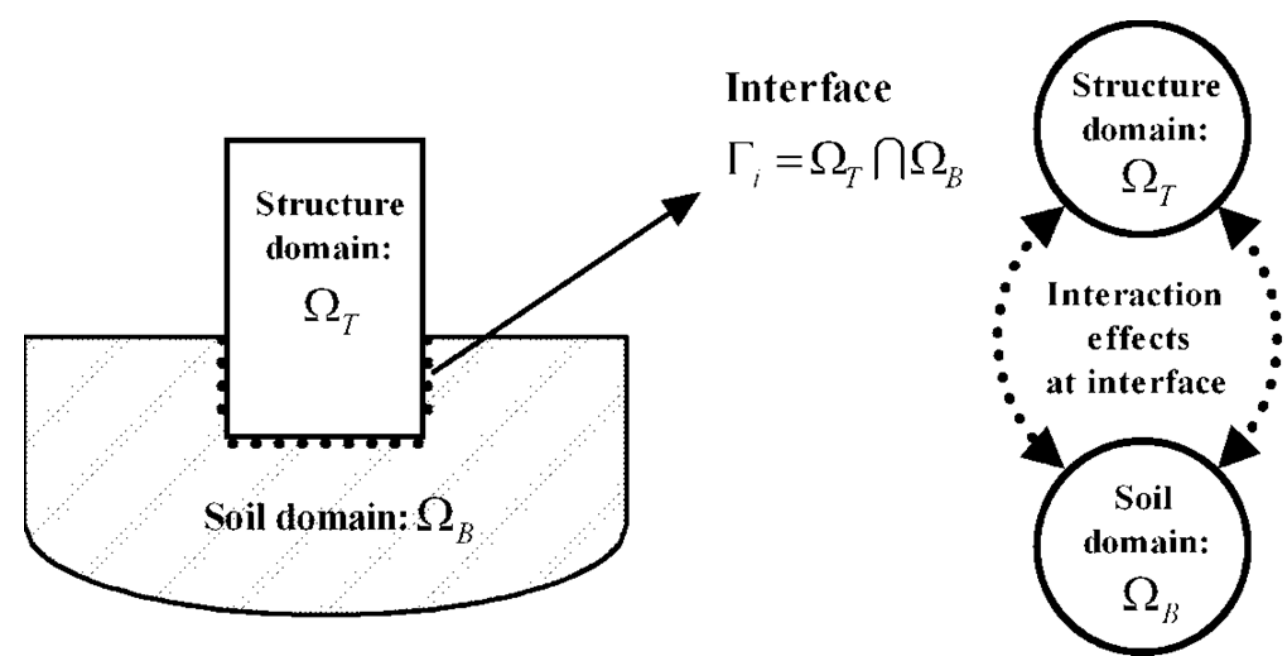


Figure 2: Communication and synchronization between ADAPTIC and ICFEP via INTERFACE

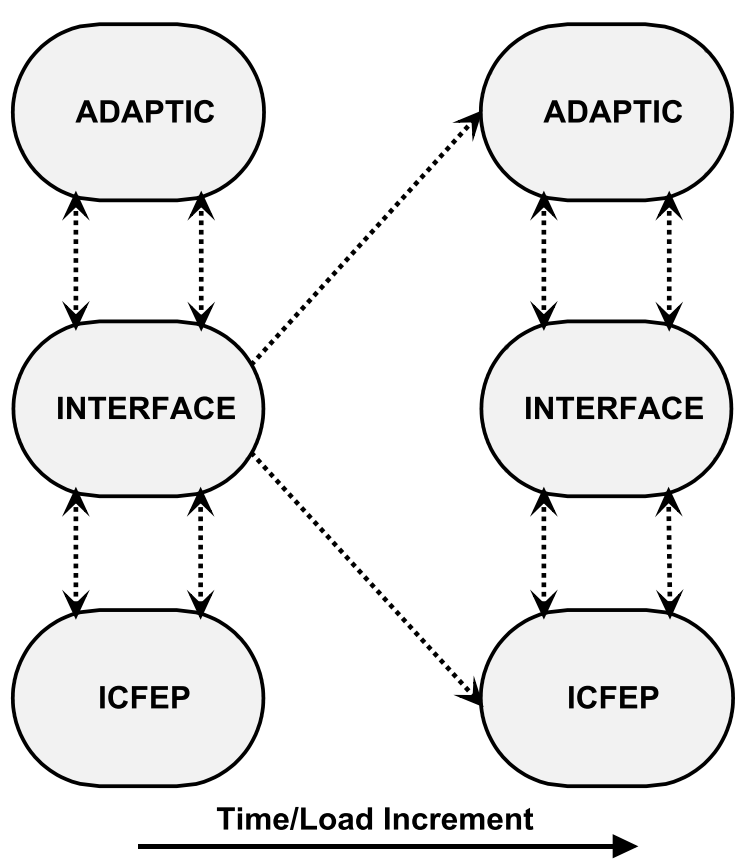


Figure 3: Data exchange structure

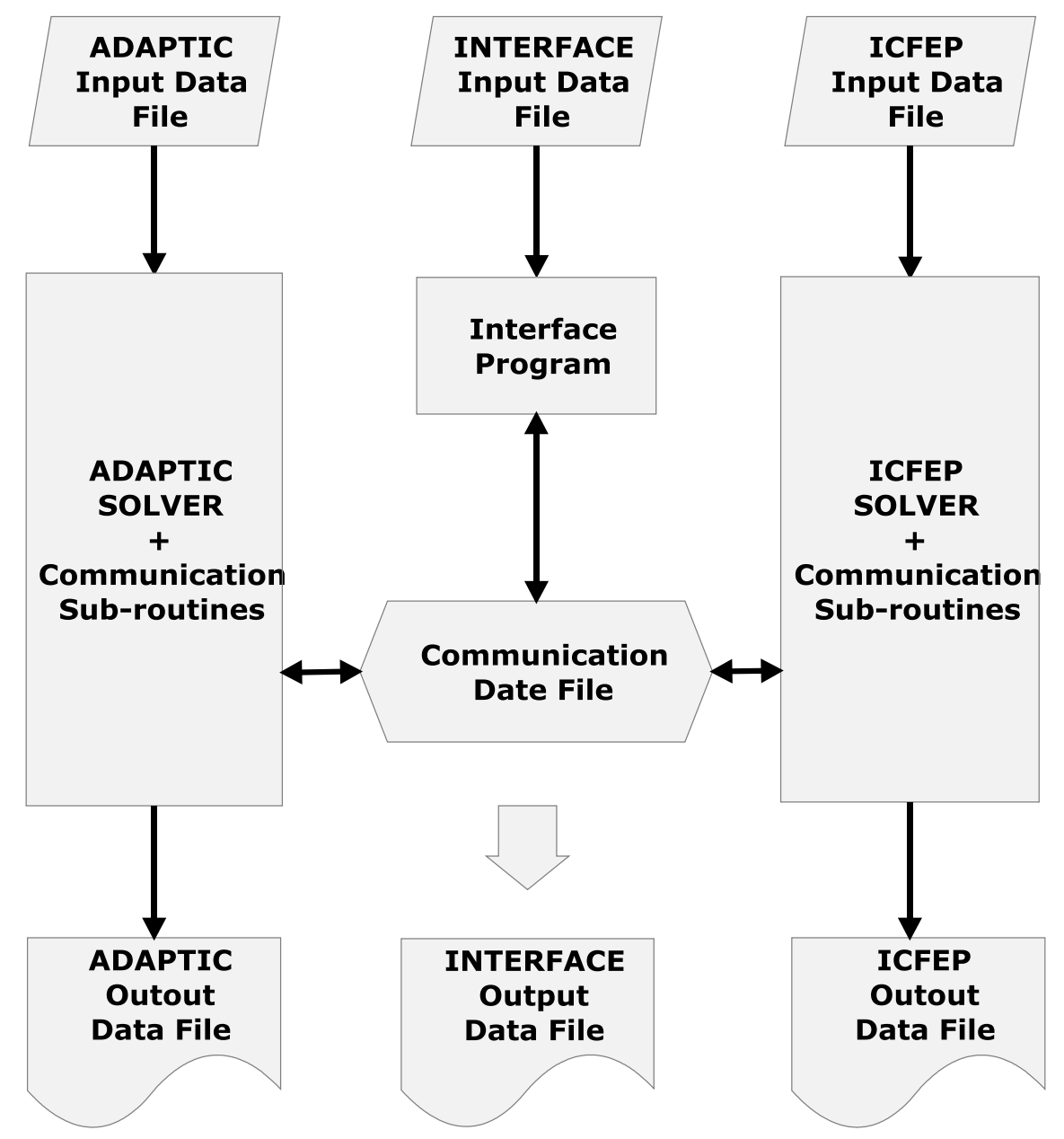


Figure 4: Schematic of the interaction sequence between INFERCAE, ADAPTIC and ICFEP

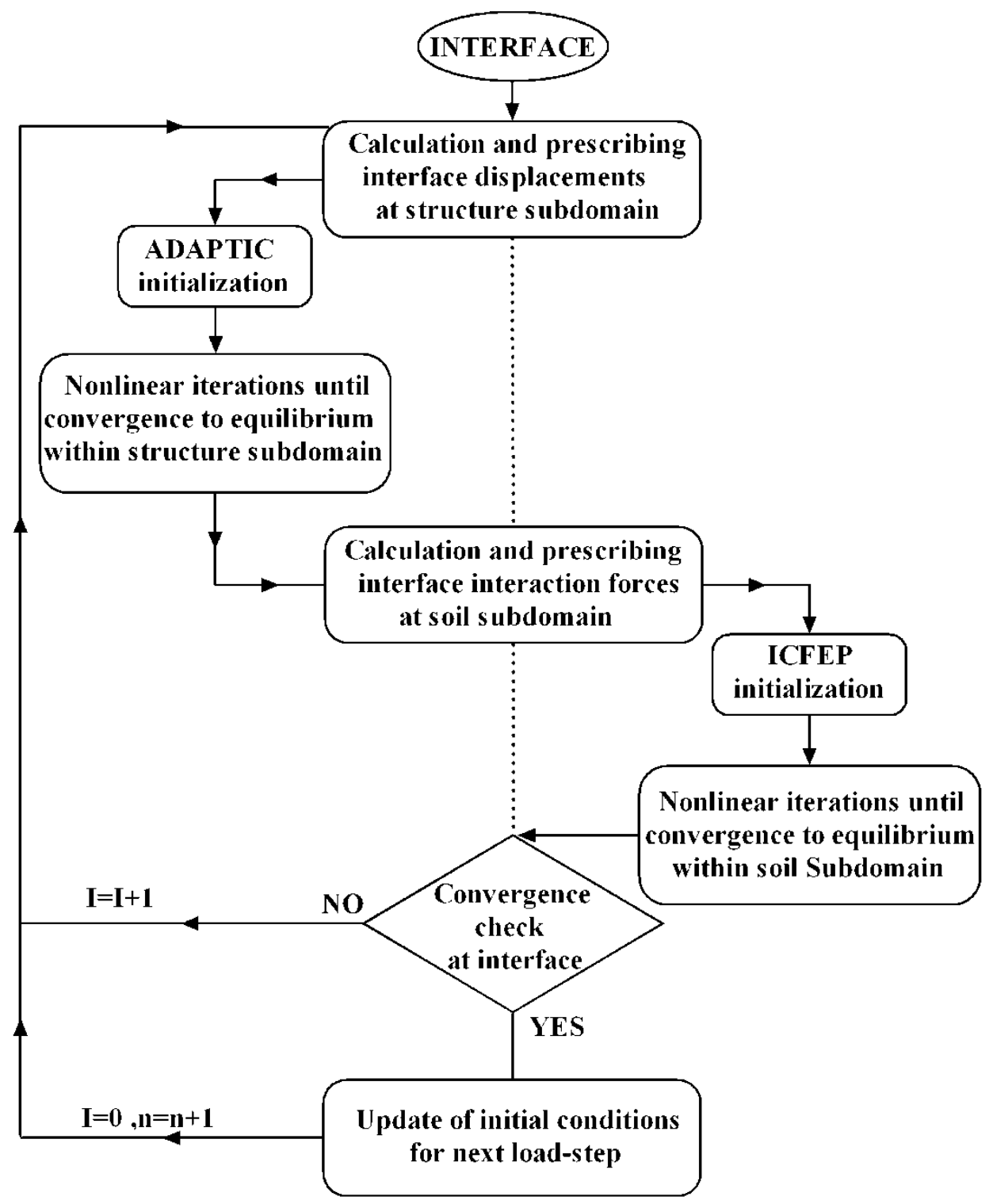


Figure 5: Linear soil-structure interaction problem

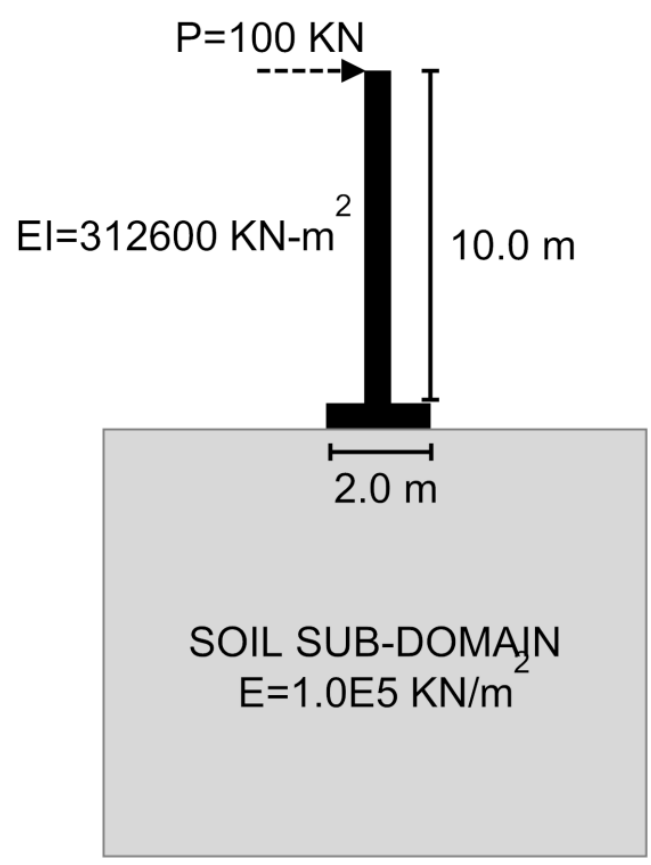


Figure 6: Monolithic vs. partitioned approach

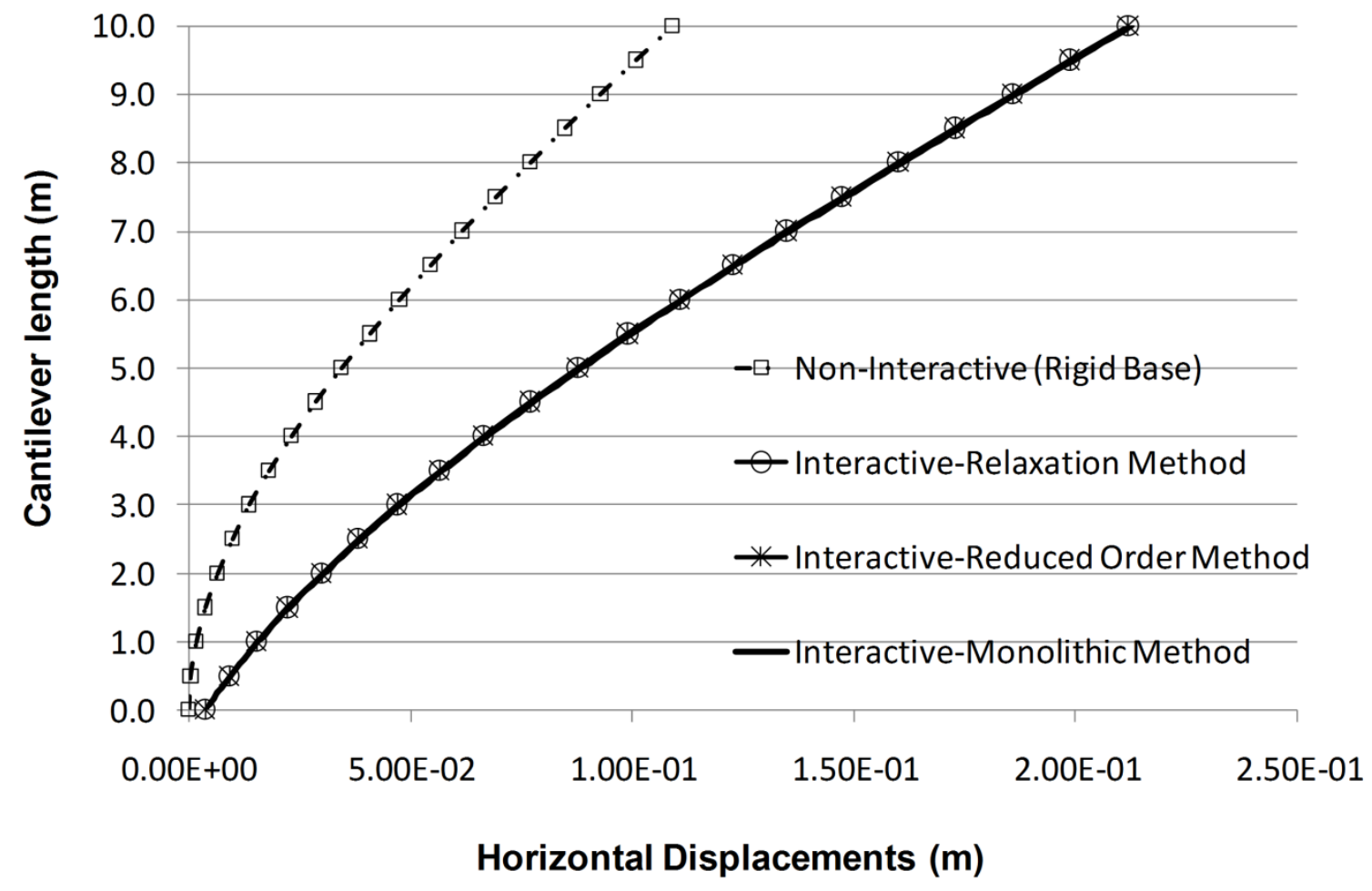


Figure 7: CPU-time of partitioned (soil/structure/interface) and monolithic approaches

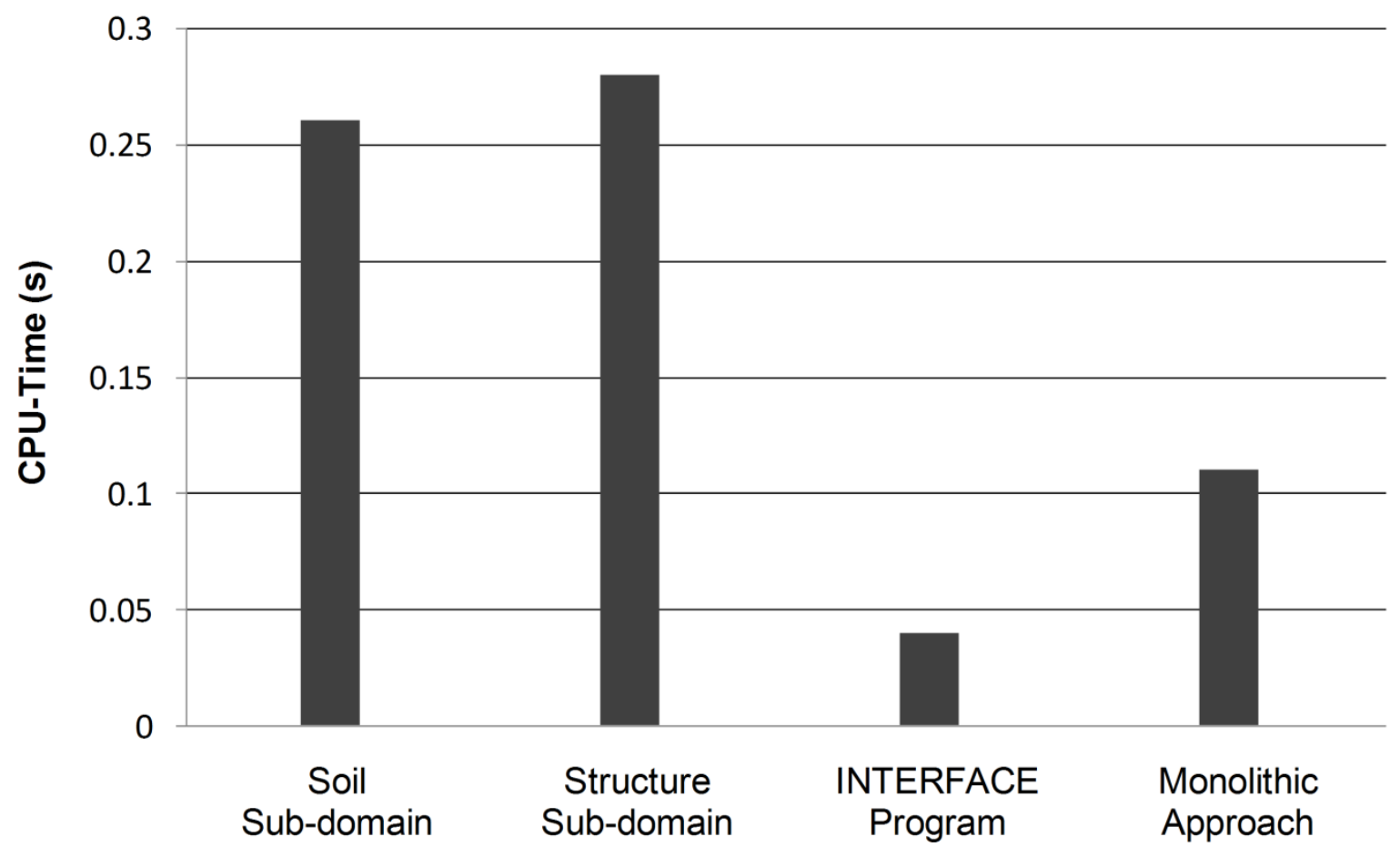


Figure 8: Plane frame resting on soil subject to ground excavation

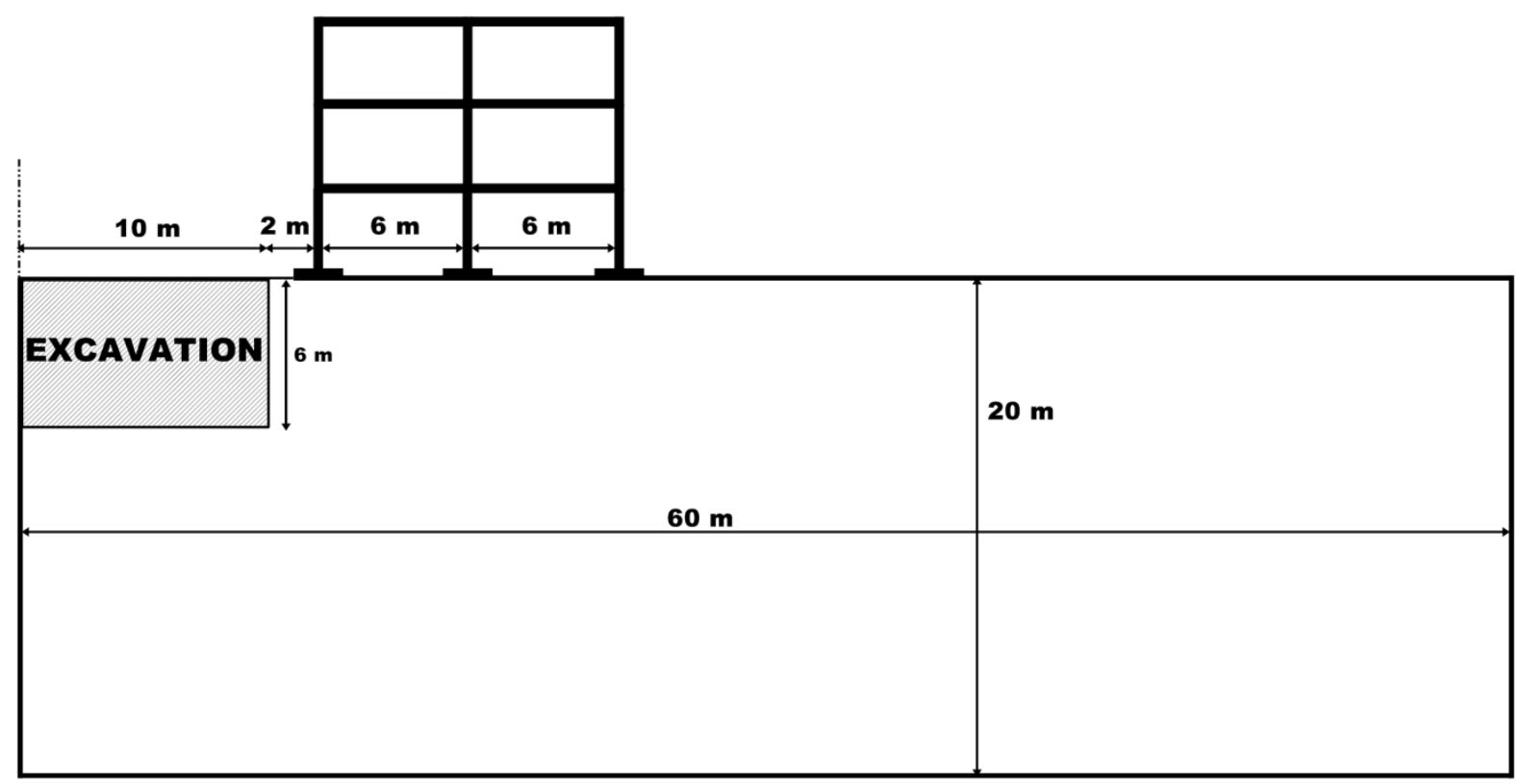


Figure 9: Plan view of the analysed building frame

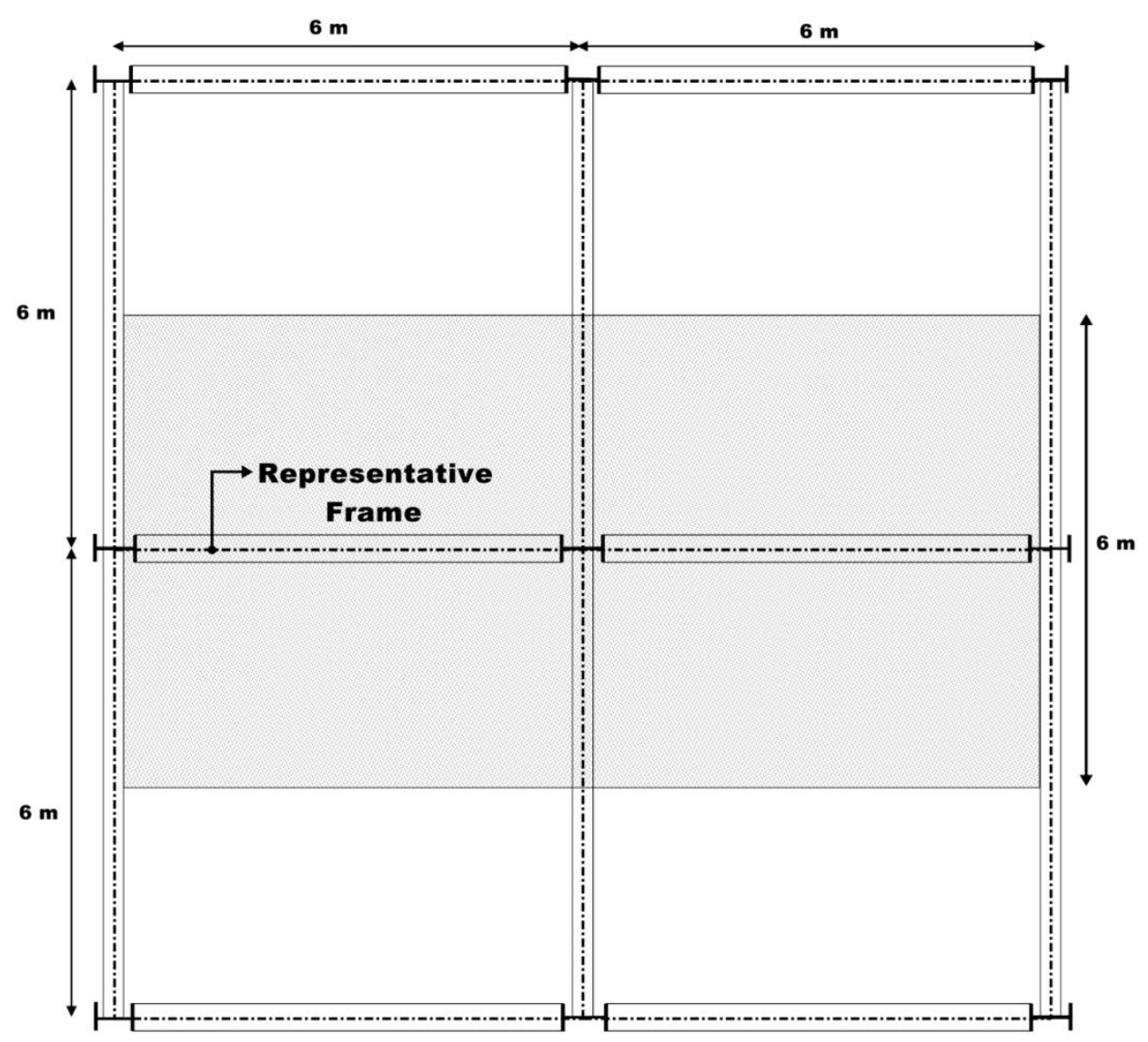


Figure 10: Geometric configuration of considered frame

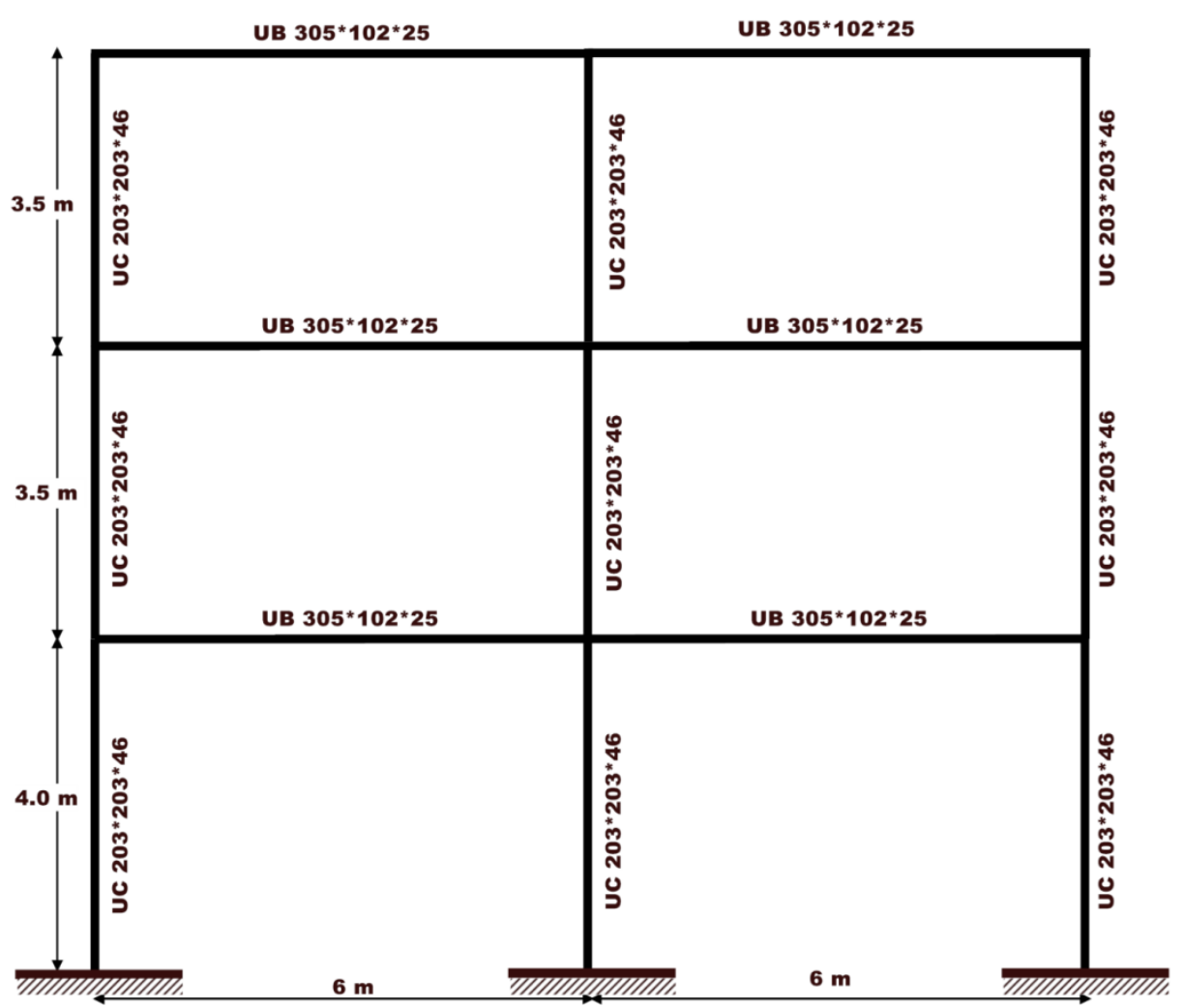


Figure 11: Influence of relaxation parameters on convergence properties

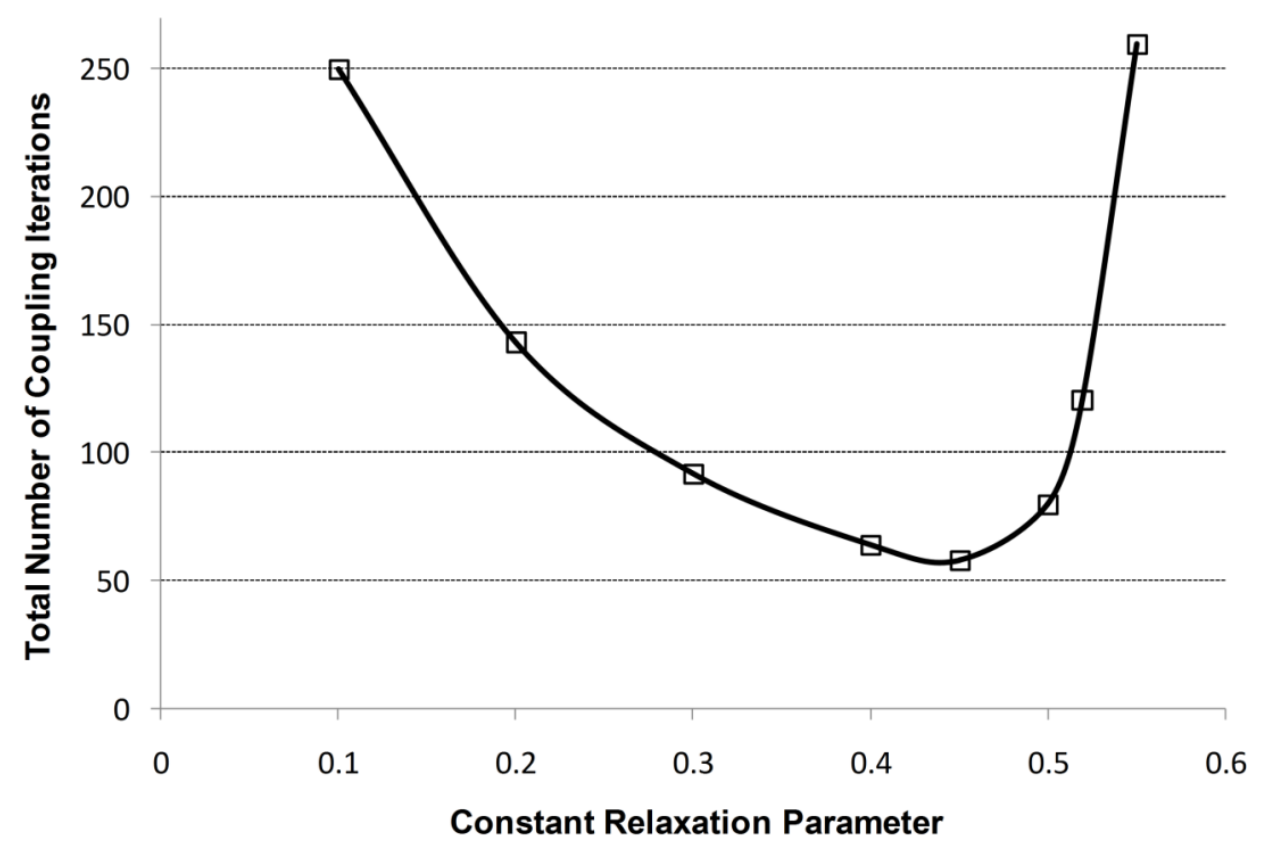


Figure 12: Error reduction for different coupling schemes for the first load increment

\section{Coupling Iteration Number}

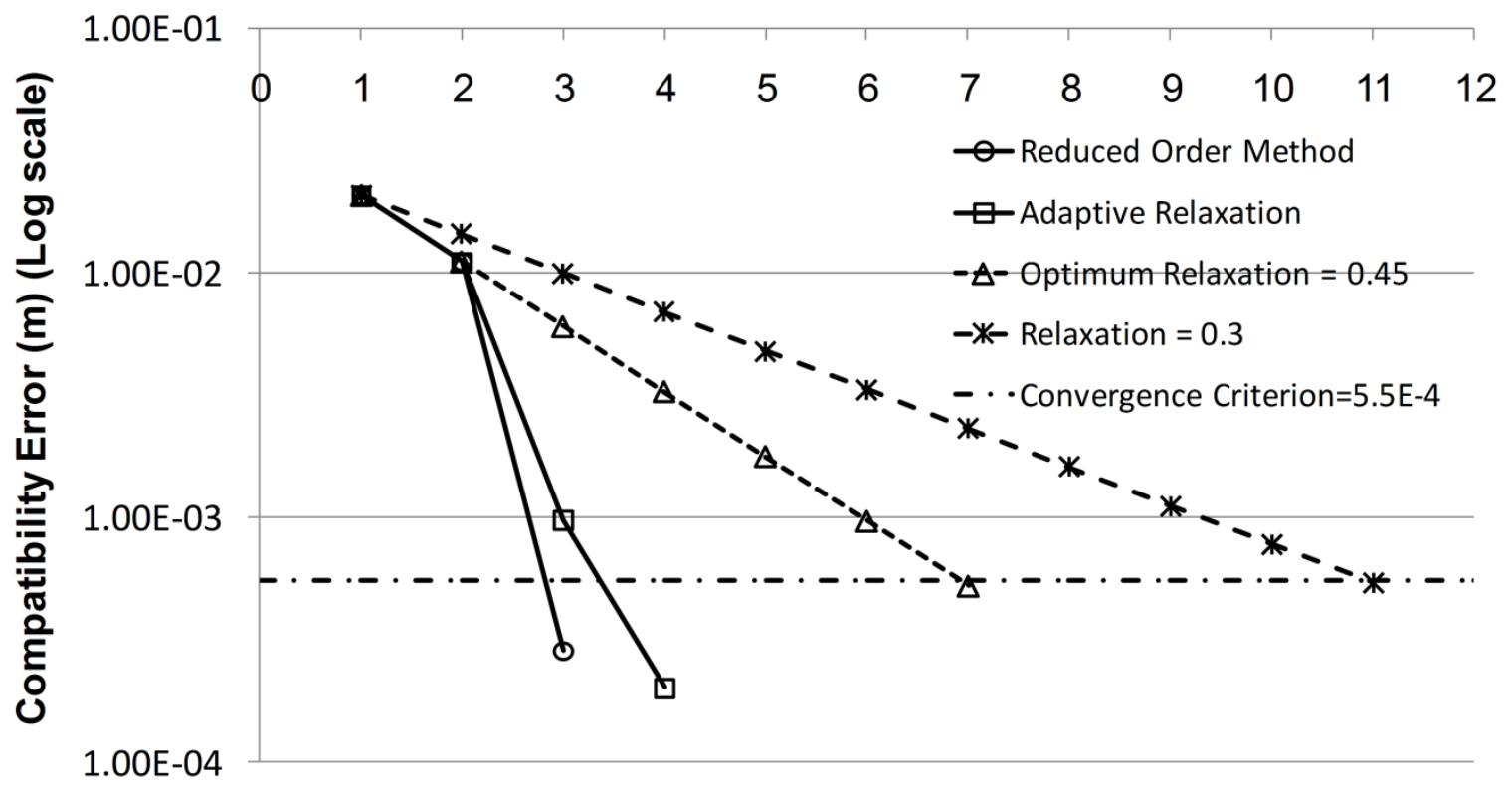


Figure 13: Error reduction for different coupling schemes for the third load increment

\section{Coupling Iteration Number}

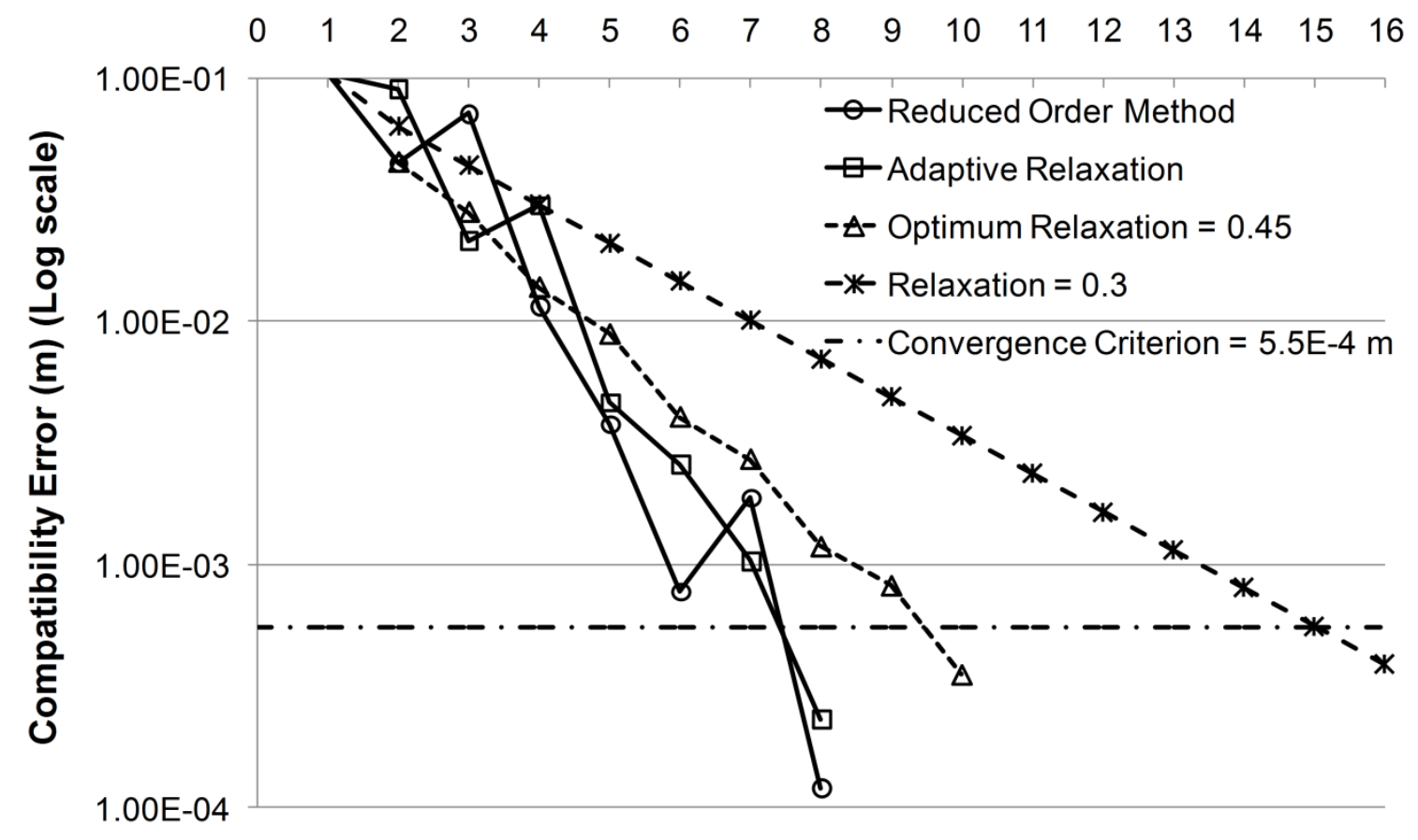


Figure 14: Error reduction for different coupling schemes for the last load increment

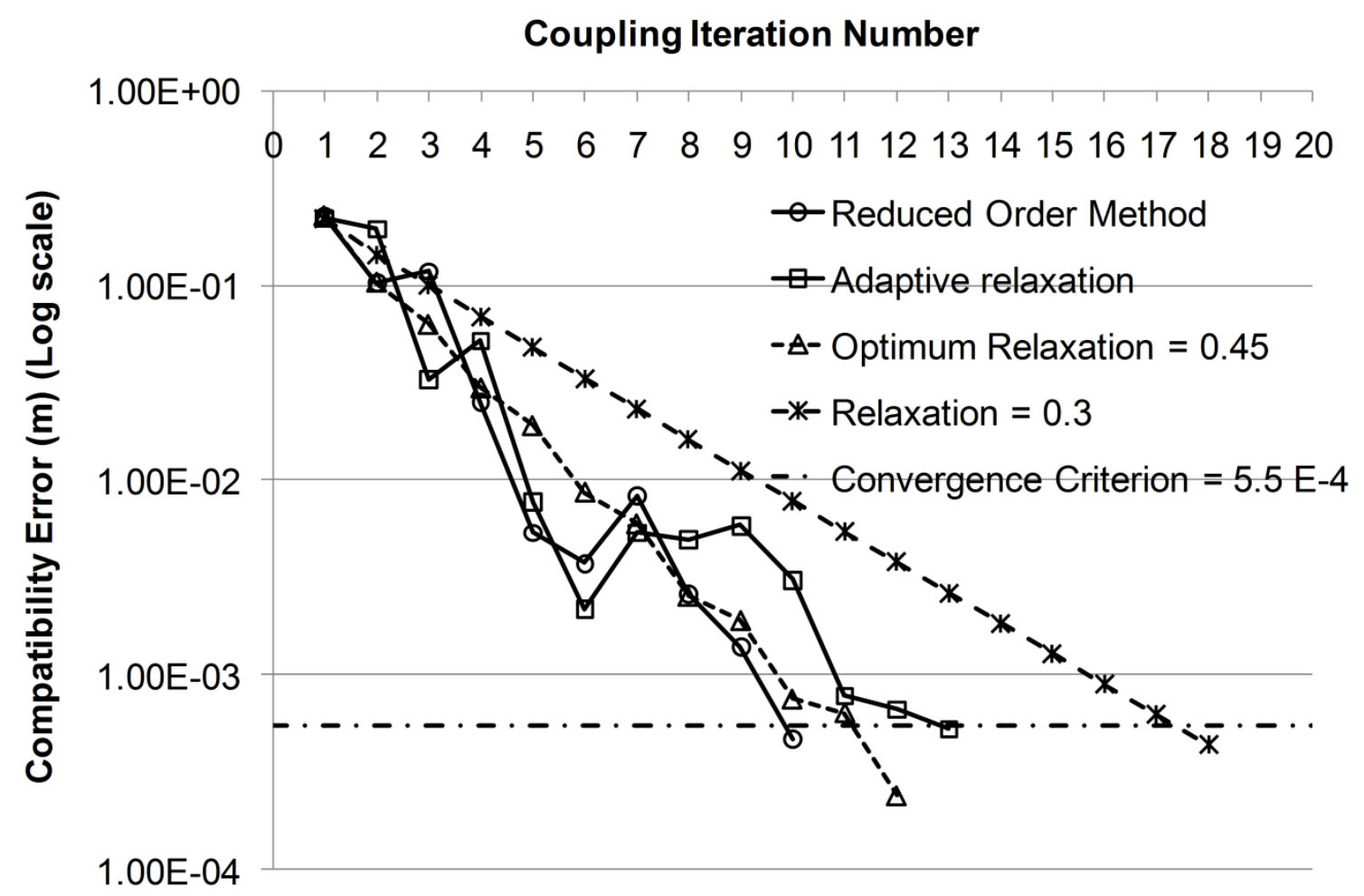


Figure 15: Convergence performance over full range of response

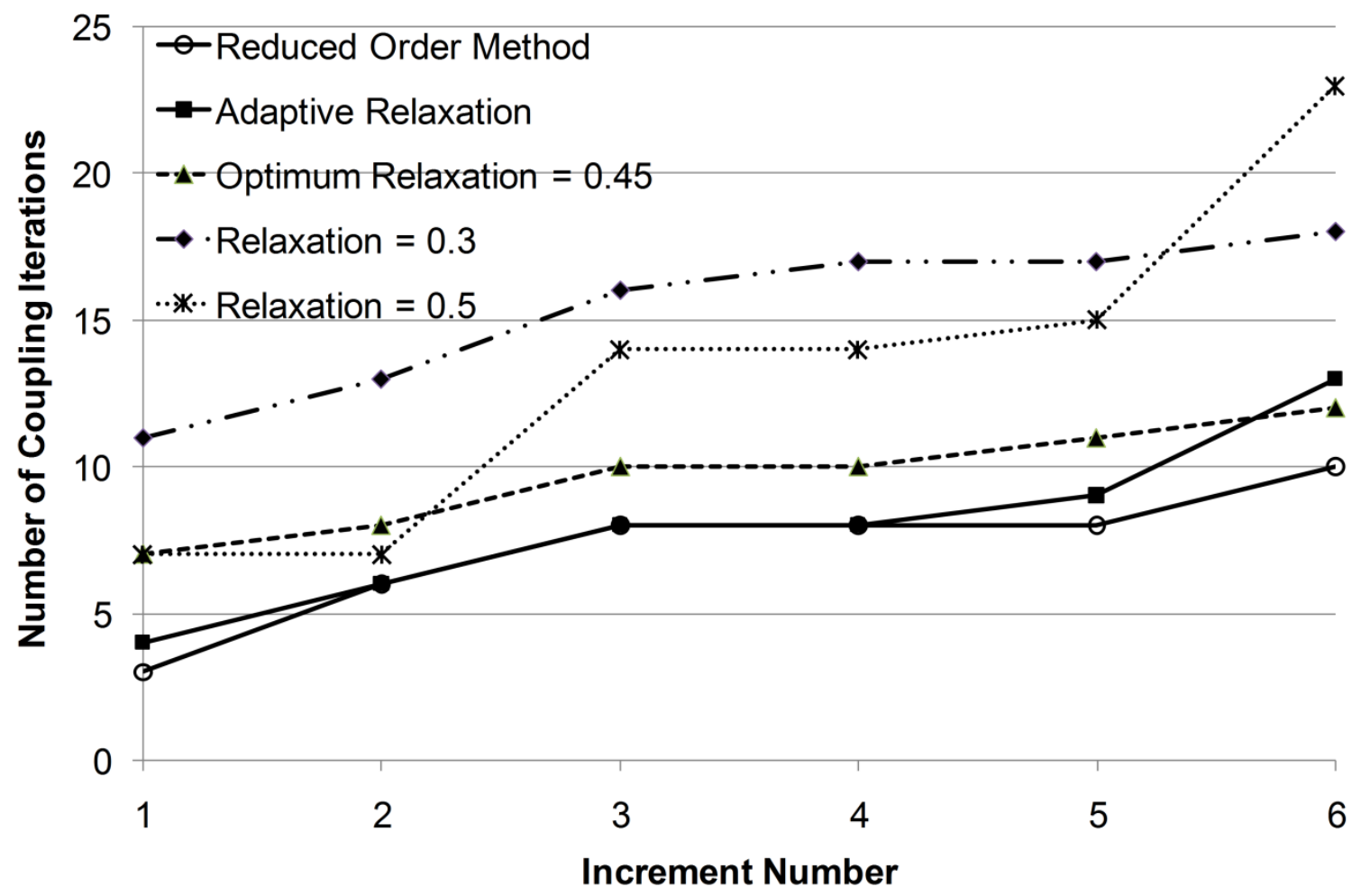


Figure 16: Vertical displacement of the soil surface

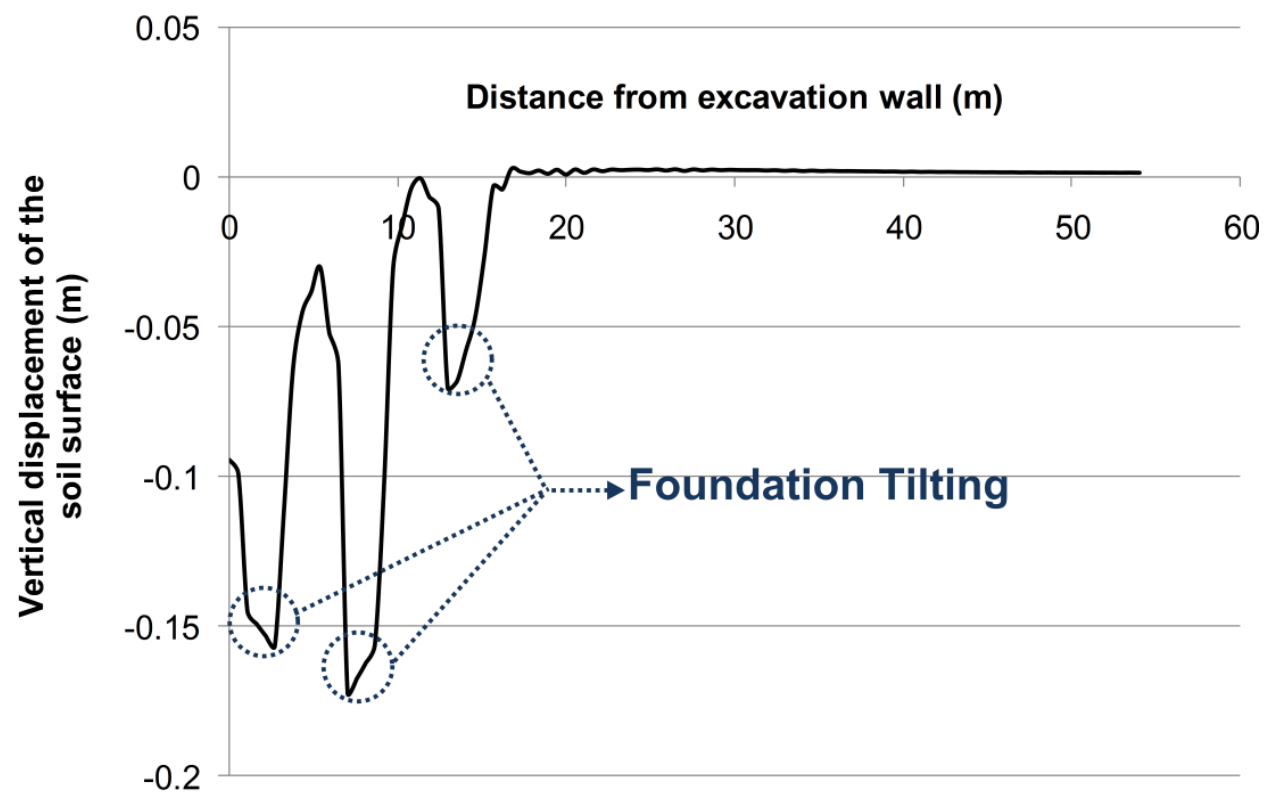


Figure 17: Horizontal displacement of the excavation

Horizontal displacement of the excavation wall (m)

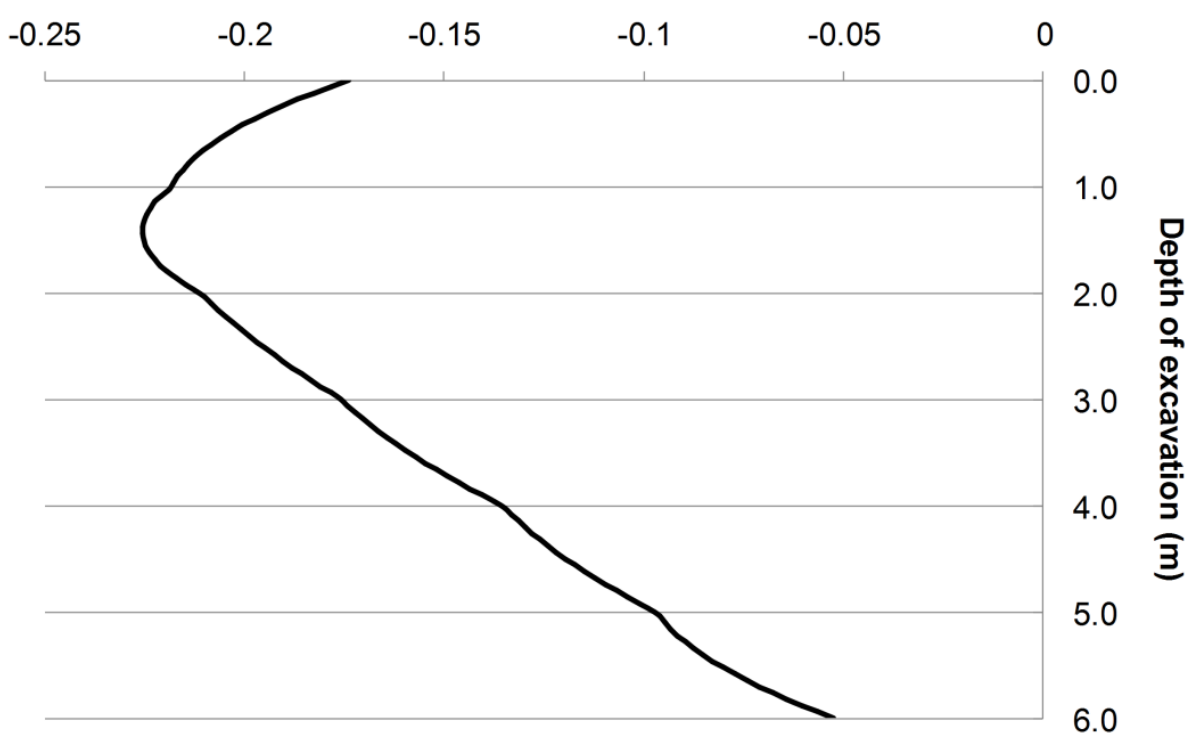


Figure 18: Vectors of displacement in soil sub-domain

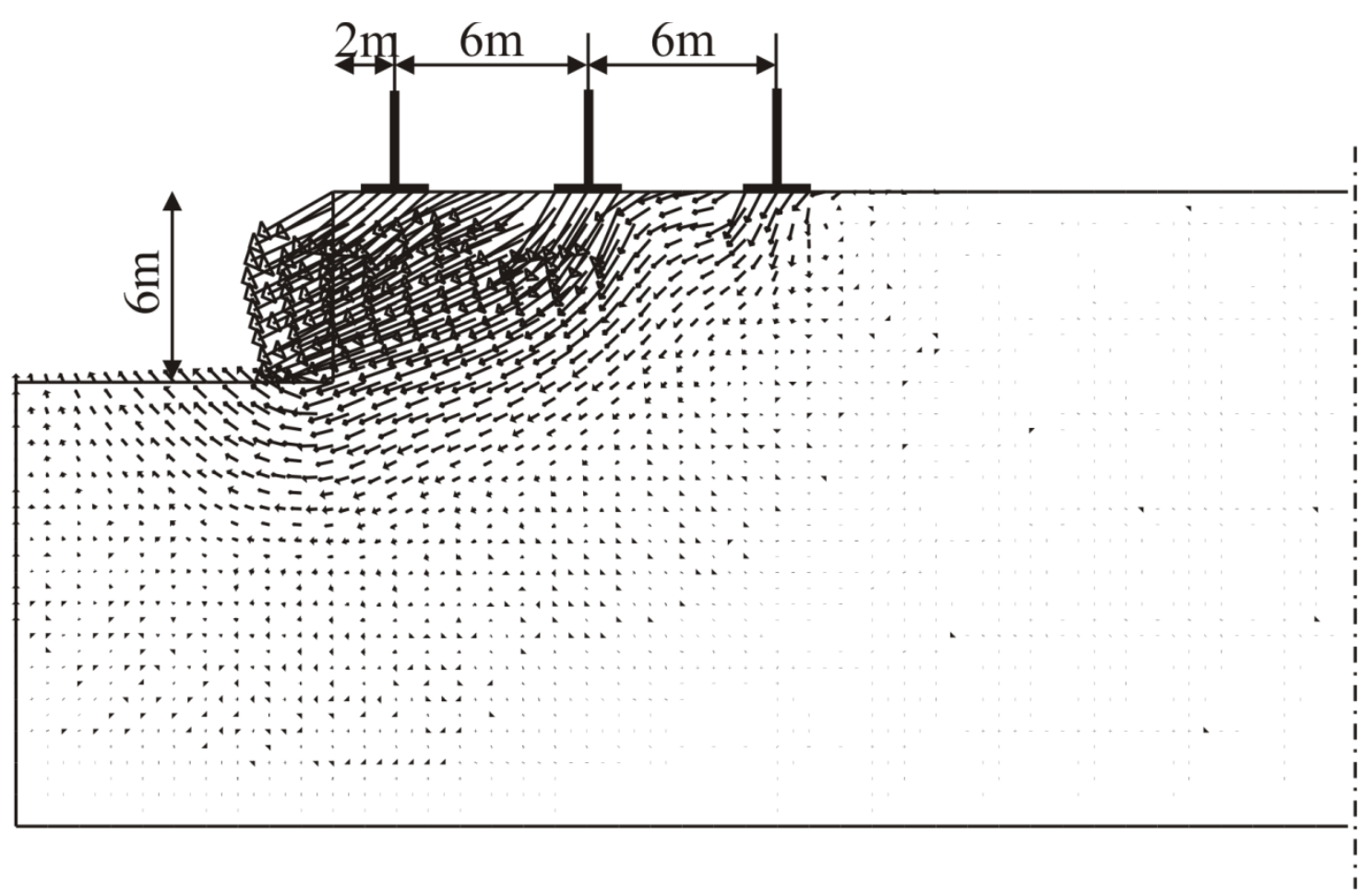


Figure 19: Contour plots of stress levels and plasticity induced in soil sub-domain

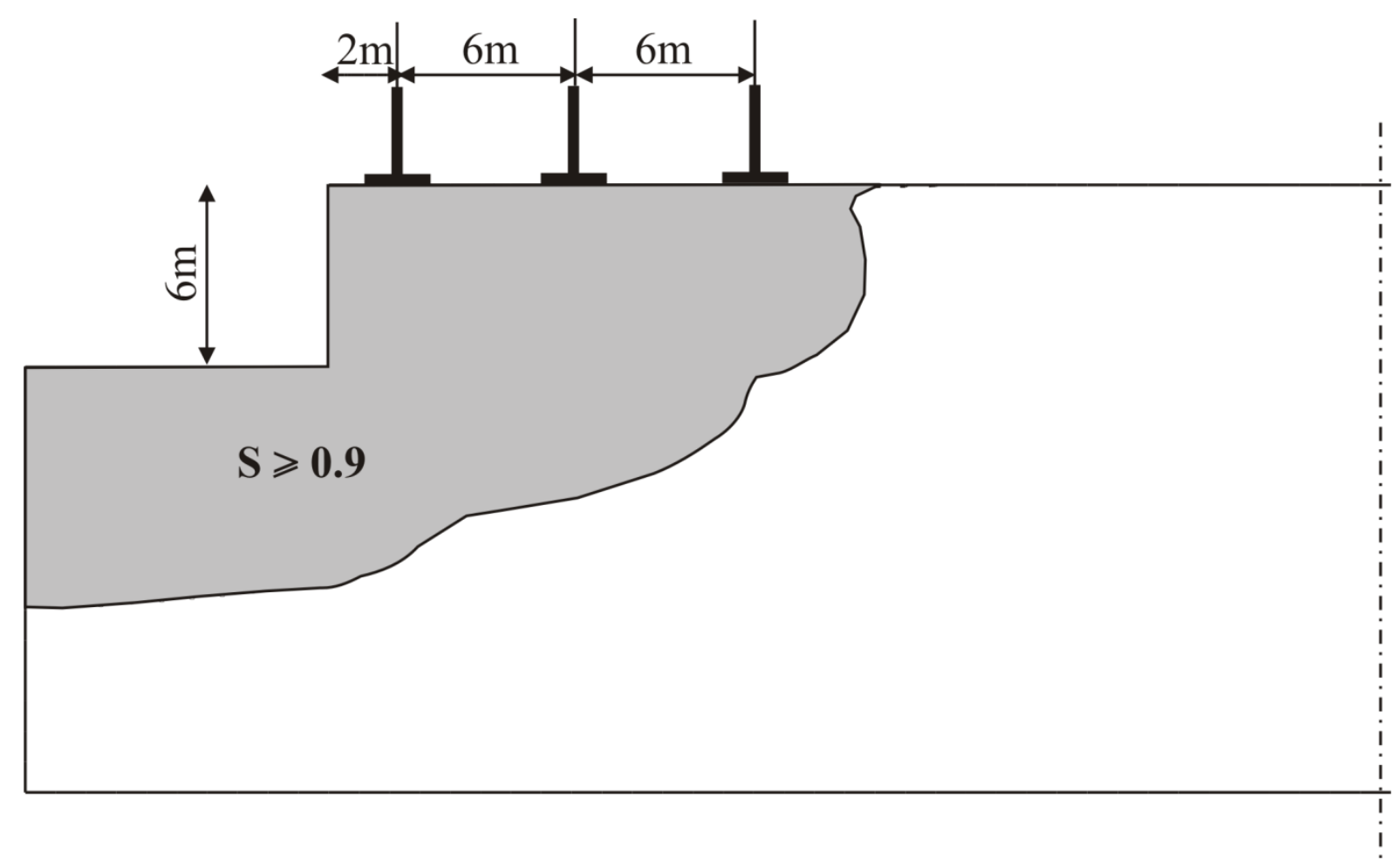


Figure 20: Deformed shape and bending moment $(\mathrm{kN}-\mathrm{m})$ of the structure sub-domain using domain decomposition approach $($ scale $=5)$

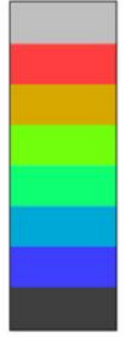

$1.750 \mathrm{e}+02$

$1.167 \mathrm{e}+02$

$5.833 e+01$

$0.000 e+00$

$-5.833 e+01$

$-1.167 e+02$

$-1.750 \mathrm{e}+02$

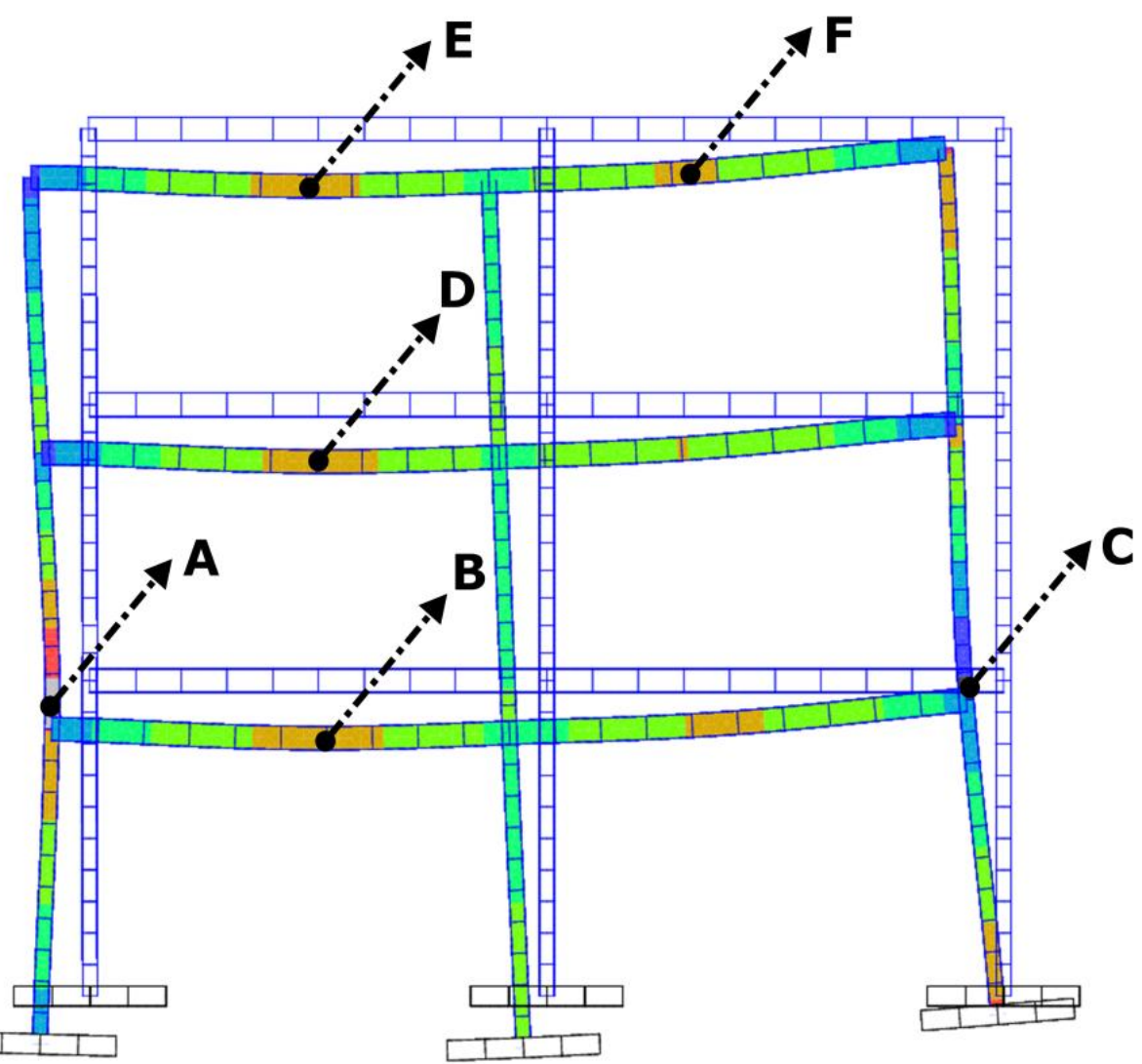


Figure 21: Deformed shape and bending moment $(\mathrm{kN}-\mathrm{m})$ of the frame using field elimination analysis via linear springs (scale $=5$ )

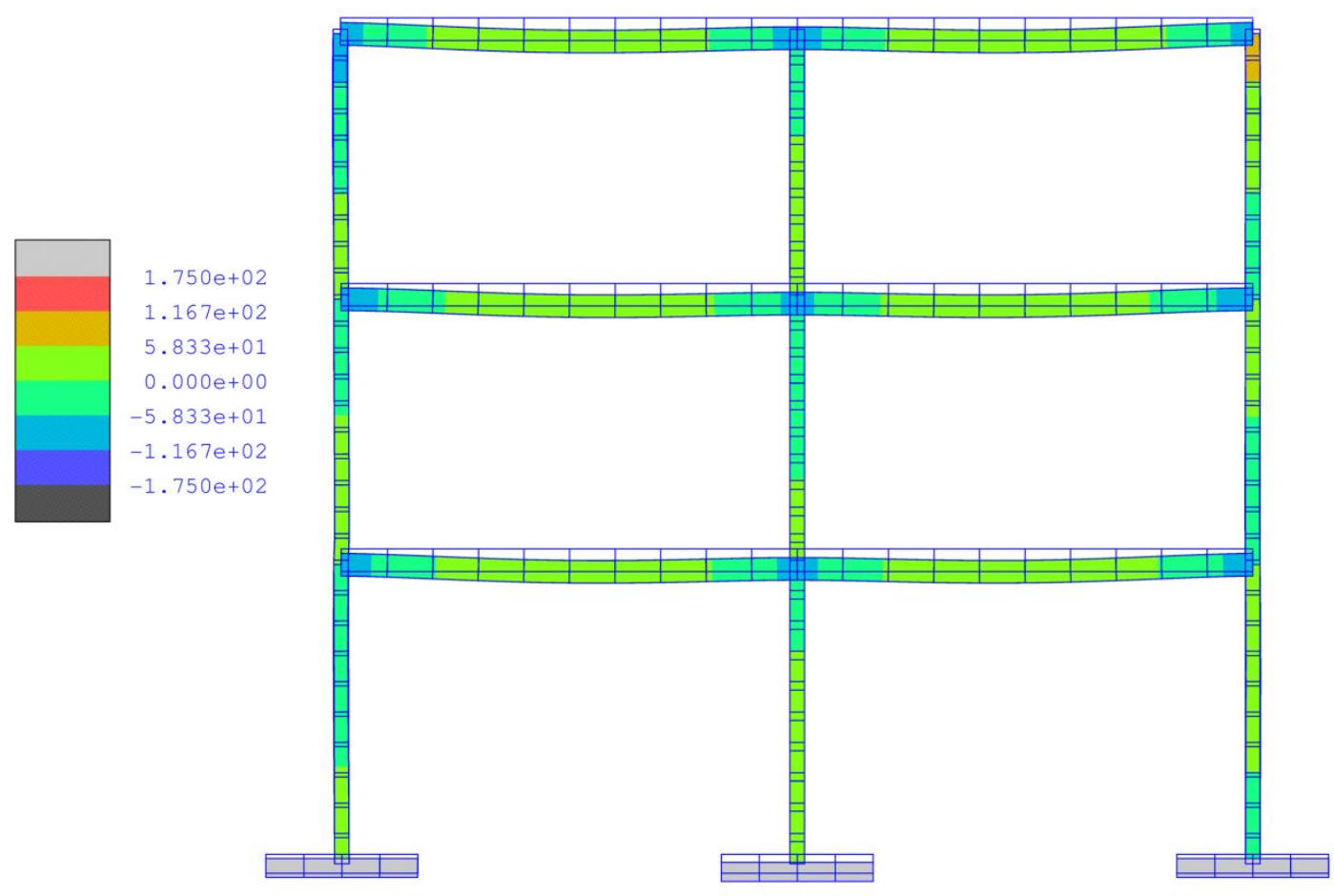

